\title{
Shedding Light On An Undiscovered Aspect That Perfectly Mimics Cosmic Acceleration
}

Karan R. Takkhi ( $\nabla$ takkhi.karan@protonmail.com )

Pune 411015, India

\section{Research Article}

Keywords: Undiscovered, supernovae, acceleration

Posted Date: September 22nd, 2021

DOl: https://doi.org/10.21203/rs.3.rs-812452/v1

License: (a) (i) This work is licensed under a Creative Commons Attribution 4.0 International License. Read Full License 


\author{
Karan R.Takkhi* \\ Pune, 411015, India \\ *E-mail: takkhi.karan@protonmail.com
}

\begin{abstract}
The comparison of redshift-distance relationship for high and low-redshift supernovae revealed the surprising transition of Universe's expansion from deceleration to acceleration. As compared to local supernovae, remote supernovae are further away than expected. The expansion rate obtained for local supernovae is higher with low redshifts as compared to the expansion rate obtained for remote supernovae with high redshifts. Since observed redshifts provide an estimate of recession/expansion velocities in order to determine the expansion rate $\left(\mathrm{km} \mathrm{s}^{-1} \mathrm{Mpc}^{-1}\right)$ of the Universe, therefore, it is very disturbing to find that low recession velocities (just $1 \%$ of speed of light) indicate a faster rate of expansion (acceleration), whereas high recession velocities (60\% of speed of light) indicate a slower rate of expansion (deceleration). In this paper I unravel an undiscovered aspect that perfectly mimics cosmic acceleration. Rather than "cosmic deceleration that preceded the current epoch of cosmic acceleration", I show that "consecutive expansion epochs of the Universe that preceded the current expansion epoch were responsible for placing remote supernovae further away than expected". As a consequence of consecutive expansion, expansion began for remote structures in preceding expansion epochs before it did for local structures in the current expansion epoch; remote supernovae are therefore not only further away than expected, but they also happen to yield a slower rate of expansion even with "superluminal expansion velocities". As a result of consecutive expansion, preceding expansion epochs appear to be decelerating as compared to the expansion epoch that succeeds them. The results obtained have been confirmed by plotting velocity-distance relationship, expansion rate vs. time relationship, expansion factor vs. time relationship, scale factor vs. time relationship, scale factor vs. distance relationship, distance-redshift relationship, and distance modulus vs. redshift relationship, moreover, deceleration parameter $\left(q_{0}\right)$ is also found to be negative $\left(q_{0}<0\right)$.
\end{abstract}

\section{INTRODUCTION}

The research conducted by the High-Z Supernova Search Team (Riess et al. 1998) and by the Supernova Cosmology Project team (Perlmutter et al. 1999) by using type Ia supernovae as standard candles resulted into a very surprising discovery that made the teams win the 2011 Nobel Prize in Physics. By comparing the brightness of the very distant, remote supernovae with the brightness of the nearby ones, remote supernovae were found to be $10 \%$ to $25 \%$ dimmer than the nearby local supernovae - a direct indication that remote supernovae were further away than expected. Based on this direct observation, a surprising feat was found being displayed by the Universe, a feat that was so extraordinary that the remarkable results obtained were not even expected. It was the remarkable discovery of Universe expanding at an accelerating rate. A research that was aimed at observing the expected deceleration of the Universe under the gravitational influence of matter was welcomed by something completely unexpected.

"By establishing the distance to the supernovae and the speed at which they are moving away from us, scientists hoped to reveal our cosmic fate. They expected to find signs that the expansion of the Universe was slowing down, which would lead to equilibrium between fire and ice. What they found was the opposite - the expansion was accelerating" (an excerpt from "Written in the stars" by The Nobel Committee for Physics The Royal Swedish Academy of Sciences)

A mysterious energy of unknown origin, having no explanation in fundamental physics and therefore rightfully coined as dark energy is considered responsible for accelerating the Universe's expansion. According to Durrer (2011), "our single indication for the existence of dark energy comes from distance measurements and their relation to redshift. Supernovae, cosmic microwave background anisotropies and observations of baryon acoustic oscillations simply tell us that the observed distance to a given redshift is larger than the one expected from the locally measured Hubble parameter".

The expansion history of the Universe is depicted by the Hubble diagram as shown in Figure 1 (plotted by using the Supernova Cosmology Project data for 580 type Ia supernovae from Union 2 (Amanullah et al. 2010) and Union 2.1 (Suzuki et al. 2012), 7 additional high-redshift type Ia supernovae discovered through the ACS (Advanced Camera for Surveys) on the Hubble Space Telescope from the GOODS (Great Observatories Origins Deep Survey) Treasury program (joint work conducted by Giavalisco et al. 2004 and Riess et al. 2004), and 1 additional very high-redshift type Ia supernova discovered with WFPC2 (Wide Field and Planetary Camera 2) on the Hubble Space Telescope (Gilliland et al. 1999)).

The observed deviation from redshift-distance linearity in Figure 1 indicates an accelerating Universe since the distances to the remote supernovae are larger than expected with respect to the nearby ones. The value of slope (or the expansion rate measured in $\mathrm{km} \mathrm{s}^{-1} \mathrm{Mpc}^{-1}$ ) is higher for the local structures and lower for the remote structures, suggesting that the Universe is accelerating now and was decelerating in the past. "A purely kinematic interpretation of the SN Ia sample provides evidence at the greater than $99 \%$ confidence level for a transition from deceleration to acceleration or, similarly, strong evidence for a cosmic jerk" (Riess et al. 2004).

By comparing the redshift-distance relationship for remote and local supernovae, cosmologists have presented groundbreaking conclusive evidence regarding the transition of Universe's expansion that favours a recent accelerated expansion and a previous decelerated expansion. "Observations of Type Ia supernovae ( $\mathrm{SNe}$ Ia) at redshift $z<1$ provide startling and puzzling evidence that the expansion of the universe at the present time appears to be accelerating" (Riess et al. 2004). It is believed that the Universe was decelerating in the past due to the gravitational attraction of matter (Riess et al. 2001, Riess 2012). "A single SN Ia at $z \approx 1.7$, SN $1997 \mathrm{ff}$, discovered with WFPC2 on the Hubble Space Telescope (HST) (Gilliland et al. 1999), provided a hint of past deceleration" (Riess et al. 2004).

Why does it appear that the Universe was decelerating in the past and is accelerating now? Why do remote supernovae appear $10 \%$ to $25 \%$ dimmer than the nearby local supernovae, thereby making us believe that they are further away than expected? Could distant supernovae be appearing dim due to intervening dust? Or could it be that those distant supernovae have different properties as compared to the nearby local supernovae? These possibilities have already been taken into account and have therefore been addressed - dust is not a factor, similarly, the brightness of local and remote supernovae differing due to property mismatch brought about by evolution is also not a factor (Riess 2000, Coil et al. 2000, Leibundgut 2001, Sullivan et al. 2003, Riess et al. 2004).

The aim of this paper is to shed light on an undiscovered aspect that mimics cosmic acceleration so perfectly well that it tricks us into believing that the Universe was decelerating in the past and is accelerating now.

\section{THE SURPRISING TRANSITION OF UNIVERSE'S EXPANSION FROM DECELERATION TO ACCELERATION: ANALYSING REMOTE AND LOCAL TYPE Ia SUPERNOVAE}

In an expanding Universe the observed redshifts provide an estimate of recession velocities. For instance, a redshift $(z)$ of 0.1 corresponds to a recession velocity of $30,000 \mathrm{~km} \mathrm{~s}^{-1}$. Once the redshifts and the distances are known (distances of type Ia supernovae estimated from their standard luminosities), the relation between redshift and distance is then used to determine the expansion rate $\left(\mathrm{km} \mathrm{s}^{-1} \mathrm{Mpc}^{-1}\right)$ of the Universe.

In Figure 1, the very high-redshift remote supernova $(z=1.7)$ yields a slope of $1.2949 \times 10^{-18} \mathrm{~m} \mathrm{~s}^{-1} \mathrm{~m}^{-1}\left(\approx 40 \mathrm{~km} \mathrm{~s}^{-1} \mathrm{Mpc}^{-1}\right)-$ consistent with the expansion rate of $46 \mathrm{~km} \mathrm{~s}^{-1} \mathrm{Mpc}^{-1}$ (Blanchard et al. 2003). On the other hand, a very low-redshift local supernova $(z=0.015166)$ that happens to fall within the linear regime of the Hubble diagram in Figure 1 yields a slope of $2.2379 \times 10^{-18} \mathrm{~m} \mathrm{~s}^{-1} \mathrm{~m}^{-1}\left(\approx 70 \mathrm{~km} \mathrm{~s}^{-1} \mathrm{Mpc}^{-1}\right)$ - consistent with the expansion rate of $72 \mathrm{~km} \mathrm{~s}^{-1} \mathrm{Mpc}^{-1}$ (Freedman et al. 2001). The comparison makes it clear that the local expansion rate is significantly higher than the remote expansion rate. 


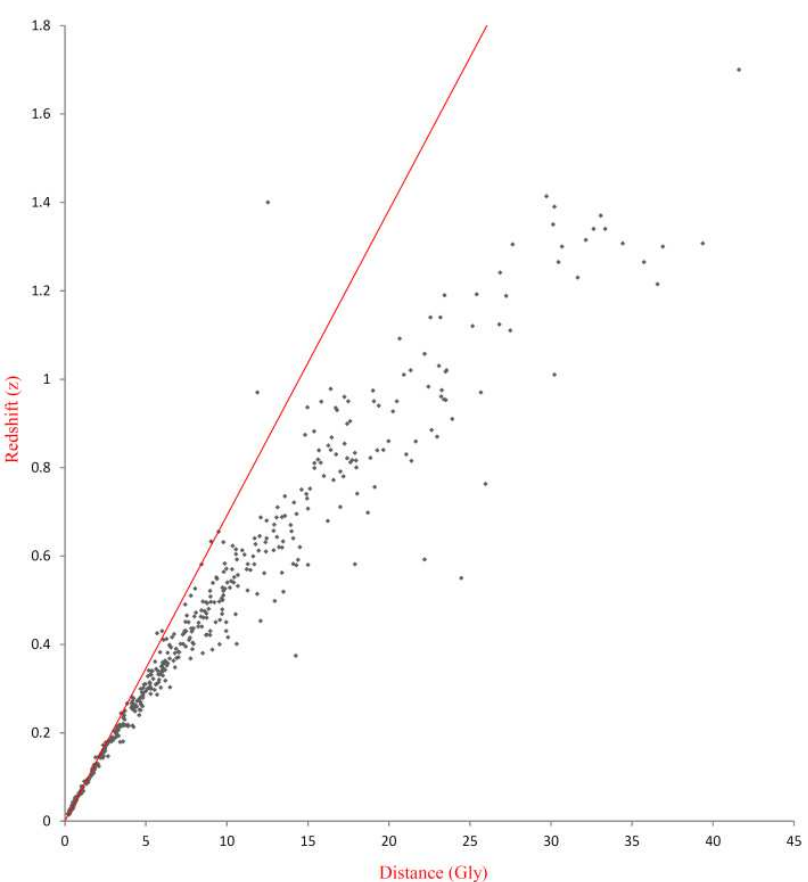

Figure 1. Redshift-distance relationship for 588 type Ia supernovae (580 type Ia supernovae plotted by using the data (Union 2 and Union 2.1) from the Supernova Cosmology Project, 7 additional high-redshift type Ia supernovae discovered through the ACS (Advanced Camera for Surveys) on the Hubble Space Telescope from the GOODS (Great Observatories Origins Deep Survey) Treasury program, and 1 additional very high-redshift type Ia supernova discovered with WFPC2 (Wide Field and Planetary Camera 2) on the Hubble Space Telescope). The red line indicates the linear redshift-distance relationship exhibited by the local structures. The deviation from linearity indicates an accelerating Universe since the distances to the remote supernovae are larger than expected with respect to the local supernovae. The slope is steeper for the local structures suggesting a faster rate of expansion (acceleration) and shallower for the remote structures, suggesting a slower rate of expansion (deceleration).

The redshift of the remote supernova is 112 times higher than the redshift of this very nearby local supernova. Since observed redshifts provide an estimate of recession velocities as shown in Figure 2, Figure 3, and Figure 4, therefore, confidently, those recession velocities, or expansion velocities corresponding to those observed high redshifts exhibited by the remote supernovae are undoubtedly much higher. Such aspect cannot be ignored particularly when recession velocities from observed redshifts due to expansion are being used to determine the expansion rate of the Universe. In fact, the unit of expansion rate $\left(\mathrm{km} \mathrm{s}^{-1} \mathrm{Mpc}^{-1}\right)$ makes it clear enough that there is a velocity and a distance component associated with the measurement of Universe's rate of expansion; it is this unit of measurement that helps us to compare the expansion rate of the remote and the local Universe in order to determine if the Universe is expanding at a slower rate, or at a faster rate.

Such high redshift of the remote supernova does not indicate in any way a low recession velocity, or a slower rate of expansion, or deceleration due to the gravitational attraction of matter! Therefore, one should explain why does a further-awaythan-expected remote supernova even with high recession velocity ends up yielding a lower value of slope (a slower rate of expansion, or deceleration) as compared to a nearby local supernova that yields a higher value of slope (a faster rate of expansion, or acceleration) even with low recession velocity?

\section{ANALYSING THE SUPERNOVA SN 1995K}

SN 1995K was the first and the most distant type Ia supernova discovered in 1995 by the High-Z Supernova Search Team. As compared to the nearby type Ia supernovae that happen to fall within the linear regime of the Hubble diagram as shown in Figure 2, SN 1995K happens to deviate from linearity as it is further away than expected. SN 1995K showed deceleration parameter $\left(q_{0}\right)=-0.6$ (Schmidt 2012). Negative value for the deceleration parameter clearly implied that the Universe was accelerating. However, additional supernovae were required by the team to confirm if the Universe was accelerating or decelerating, and, it was only through further observations of additional type Ia supernovae at even larger distances that confirmed an accelerating Universe (Figure 3).

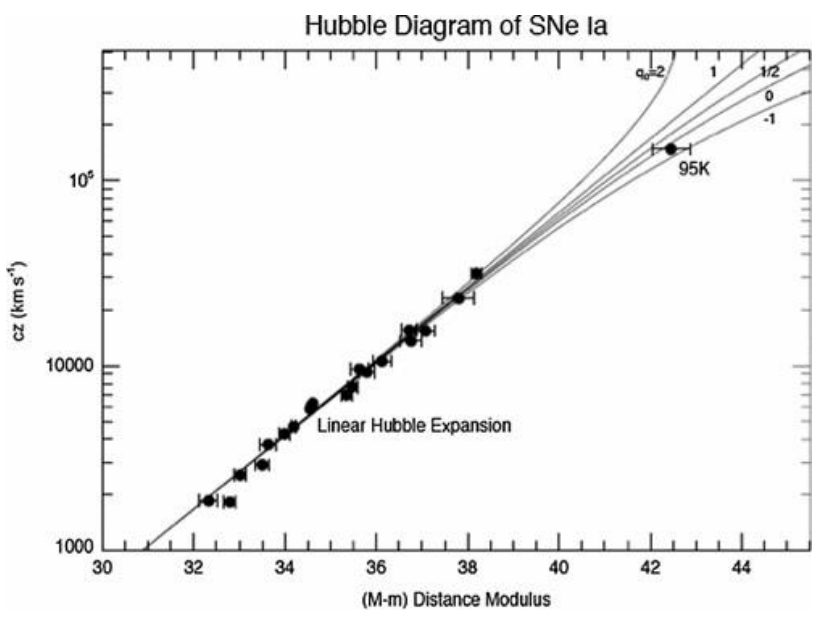

Figure 2. Velocity-distance relationship (Hubble Diagram of SNe Ia) showing SN 1995K at a redshift $(z)$ of 0.479 from the proposal put forward by the High-Z Supernova Search Team. Credit: Schmidt B. P., Reviews of Modern Physics, vol. 84, 1151, page 1158, year 2012, reprinted with permission, Copyright (2012) American Physical Society. https://doi.org/10.1103/RevModPhys.84.1151

SN $1995 \mathrm{~K}$ as plotted in Figure 2 was the most distant type Ia supernova discovered back in 1995 by the High-Z Team $(\mathrm{D}=$ 9.7211 Gly, $z=0.479)$ - this yields a slope of $1.5617 \times 10^{-18}$ $\mathrm{m} \mathrm{s}^{-1} \mathrm{~m}^{-1}\left(\approx 50 \mathrm{~km} \mathrm{~s}^{-1} \mathrm{Mpc}^{-1}\right)$. Nearby local supernovae that happen to fall within the linear regime of the Hubble diagram have also been plotted; consider one such local supernova $(\mathrm{D}=$ $0.4604 \mathrm{Gly}, z=0.0333$ ) - this yields a slope of $2.2925 \times 10^{-18}$ $\mathrm{m} \mathrm{s}^{-1} \mathrm{~m}^{-1}\left(\approx 70 \mathrm{~km} \mathrm{~s}^{-1} \mathrm{Mpc}^{-1}\right)$. The comparison of expansion rate $\left(\mathrm{km} \mathrm{s}^{-1} \mathrm{Mpc}^{-1}\right)$ for these supernovae shows that SN 1995K is expanding at a slower rate (decelerating) as compared to this nearby supernova obeying the linear Hubble expansion.

Since observed redshift is providing an estimate of recession velocity as shown here in Figure 2 itself, therefore, suggesting that SN $1995 \mathrm{~K}$ is expanding at a slower rate as compared to the nearby supernova sounds completely counterintuitive - the recession velocity of SN $1995 \mathrm{~K}$ is 14.38 times higher than the recession velocity of the nearby supernova that obeys the linear Hubble expansion, therefore, how can one justify this scientifically that SN $1995 \mathrm{~K}$ is expanding at a slower rate as compared to a local supernova?

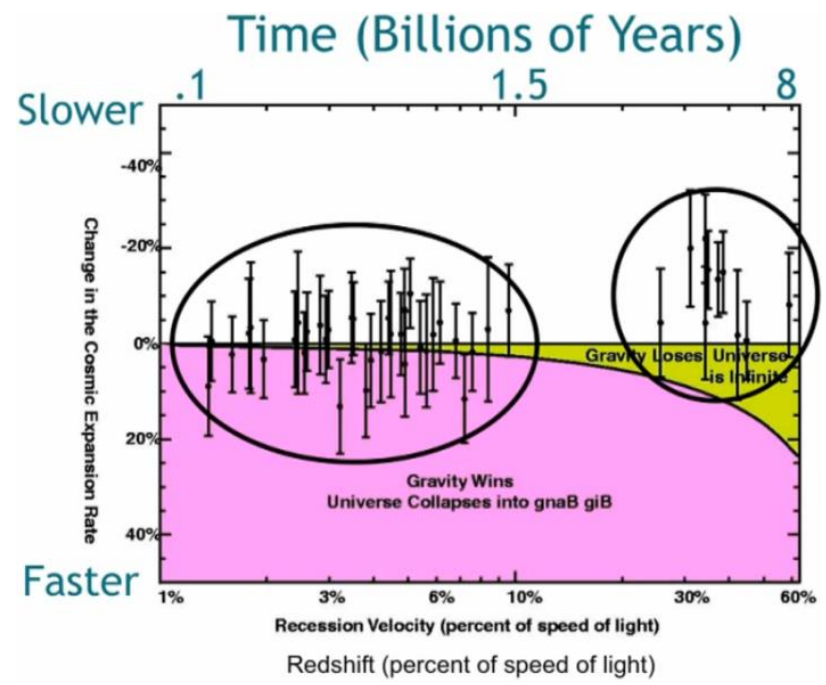

Figure 3. Observations of additional type Ia supernovae by the High-Z Supernova Search Team. The plot confirmed the result that the Universe is accelerating - remote supernovae are expanding at a slower rate (decelerating), whereas local supernovae are expanding at a faster rate (accelerating). Credit: High-Z Supernova Search Team.

Figure 3 depicts the result of additional type Ia supernovae observations at even larger distances carried out by the High-Z Team that confirmed Universe's acceleration. Distant supernovae were dimmer than expected (as they were further away than expected) and the expansion rate for them was found to be lower than the expansion rate for the nearby supernovae.

Figure 3 clearly shows the transition of Universe's expansion from deceleration to acceleration - Universe was expanding slowly in the past (decelerating remotely) and is expanding faster now (accelerating locally). 
However, if we look at the observed redshifts that provide an estimate of recession velocities as shown in Figure 3, then there seems to be a conundrum, it is very disturbing to find that local recession velocities ranging from just $1 \%$ to $10 \%$ of speed of light indicate a faster rate of expansion (acceleration), whereas remote recession velocities ranging from $30 \%$ to $60 \%$ of speed of light indicate a slower rate of expansion (deceleration).

Intriguingly, why is it that a remote object which happens to be further away than expected is yielding a lower value of slope (a slower rate of expansion, or deceleration) even with high recession velocity as compared to a nearby local object that is yielding a higher value of slope (a faster rate of expansion, or acceleration) even with low recession velocity? Such intriguing attribute is also encountered while taking into account the following plot.

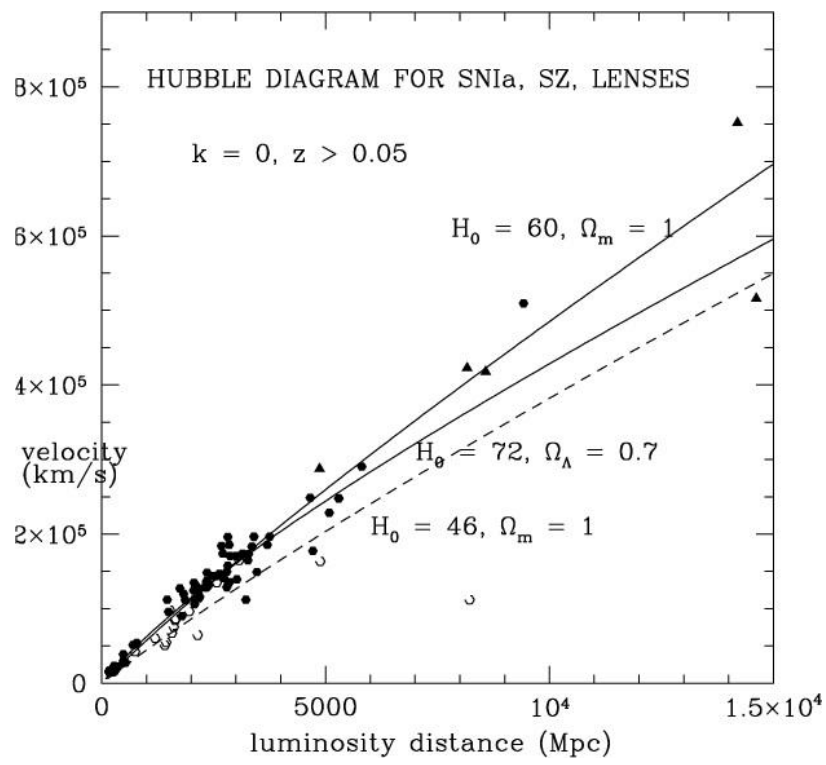

Figure 4. "Velocity versus luminosity-distance for type Ia supernovae (filled circles), $\mathrm{S}-\mathrm{Z}$ clusters (open circles) and gravitational lens timedelay systems (filled triangles), with z $>0.05$ ". Credit: Blanchard A., et al., A\&A, vol. 412, 35, page 39, year 2003, reproduced with permission (c) ESO. https://doi.org/10.1051/0004-6361:20031425

Figure 4 illustrated from Blanchard et al. (2003) shows remote measurement yielding an expansion rate of $46 \mathrm{~km} \mathrm{~s}^{-1}$ $\mathrm{Mpc}^{-1}$ which is significantly lower than the local measurement of $72 \mathrm{~km} \mathrm{~s}^{-1} \mathrm{Mpc}^{-1}$ obtained from the Hubble Key Project determination (Freedman et al. 2001). The expansion rate measured for local objects with low recession velocities is significantly greater than the expansion rate measured for remote objects with high recession velocities.

"It has been noted by Zehavi et al. (1998) that the SNe Ia out to $7000 \mathrm{~km} \mathrm{~s}^{-1}$ exhibit an expansion rate that is $6 \%$ greater than that measured for the more distant objects" (Riess et al. 1998). One might say that local void is expanding faster than the remote expansion rate. According to Riess et al. (1998), "In principle, a local void would increase the expansion rate measured for our low-redshift sample relative to the true, global expansion rate. Mistaking this inflated rate for the global value would give the false impression of an increase in the lowredshift expansion rate relative to the high-redshift expansion rate". However, according to Riess et al. (1998), "only a small fraction of our nearby sample is within this local void, reducing its effect on the determination of the low-redshift expansion rate". Furthermore, the reanalysis carried out (Riess et al. 1998) by discarding the seven SNe Ia within $7000 \mathrm{~km} \mathrm{~s}^{-1}(108 \mathrm{Mpc}$ for $65 \mathrm{~km} \mathrm{~s}^{-1} \mathrm{Mpc}^{-1}$ ) ruled out the possibility of local void and confirmed cosmic acceleration.

Anyway, the recession velocities of local structures are not high as compared to the whopping recession velocities of remote structures, in other words, the recession velocities of local structures are not high to justify a faster rate of expansion (acceleration) as compared to the recession velocities of remote structures that are not low to justify a slower rate of expansion (deceleration).

The key point is, remote structures are not only further away than expected, but they also happen to yield a slower rate of expansion even with high recession velocities as compared to the faster rate of expansion for the local structures even with low recession velocities.
Here the observed redshift has clearly been interpreted as recession velocity of $7000 \mathrm{~km} \mathrm{~s}^{-1}$ by the researchers to determine the expansion rate of the local objects $\left(65 \mathrm{~km} \mathrm{~s}^{-1}\right.$ $\mathrm{Mpc}^{-1}$ (7000 km s$\left.{ }^{-1} / 108 \mathrm{Mpc}\right)$ ); the researchers then compare this local expansion rate with the expansion rate of more distant objects to find that the expansion rate for local objects is $6 \%$ greater than the expansion rate measured for the more distant objects.

Since quantities having the same units can only be compared together, therefore, it would be very prudent to consider that the redshifts of the more distant objects might have also been interpreted in terms of recession velocities by the researchers to compare the expansion rate of local and remote structures. Therefore, why is it that an object with high recession velocity is not only further away than expected, but it is also yielding a lower value of slope (or a slower rate of expansion, thereby suggesting deceleration) as compared to a nearby object with low recession velocity?

\section{AN UNDISCOVERED ASPECT}

If there can be an epoch of "cosmic deceleration that preceded the current epoch of cosmic acceleration" (Riess et al. 2004), then there can also be epochs of "consecutive expansion that preceded the current epoch of cosmic expansion".

It remains undiscovered that an object that begins expanding before as a consequence of consecutive expansion will not only be further away than expected, but it will also be yielding a lower value of slope (or a slower rate of expansion) even with high recession velocity as compared to an object with low recession velocity that begins expanding comparatively later.

Such interpretation does not place an observer in any special or privileged position within the Universe, because, to an observer in any expansion epoch, preceding expansion epochs will appear to be decelerating, whereas the observer's current epoch of cosmic expansion that happens to succeed the preceding expansion epochs will appear to be accelerating.

Logically, an object that begins expanding before has an utmost probability of being further away than expected; the observational fact, that such object, which happens to be further away than expected, yields a lower value of slope (a slower rate of expansion, or deceleration) even with high recession velocity as compared to an object with low recession velocity is the most compelling evidence in favour of this undiscovered aspect.

Plotting together the high-recession-velocity remote structures that began expanding before and the low-recession-velocity local structures that began expanding comparatively later causes the Hubble diagram to deviate from linearity, whereas the comparison of slope and thus the expansion rate obtained from such objects causes the high-recession-velocity remote structures to appear as if they are receding slower than expected as compared to the low-recession-velocity local structures.

It must be noted that even with high recession velocity, an object that begins expanding before will never yield a value of slope, or the expansion rate that is higher than the value of slope, or the expansion rate for an object with low recession velocity that begins expanding comparatively later. Comparing the slope and thus the expansion rate of such objects results into the apparent transition of Universe's expansion from deceleration to acceleration - an object with high recession velocity that began expanding before will be further away than expected and will appear to be decelerating, whereas an object with low recession velocity that began expanding comparatively later will appear to be accelerating. It is merely this comparison that makes it appear that the Universe is accelerating now even with low recession velocities and was decelerating in the past even with high recession velocities. Requiring mysterious dark energy of unknown origin to explain this apparent transition of Universe's expansion from deceleration to acceleration would inevitably complicate things to an unimaginable extent.

\section{A SIMPLE NUMERICAL PROOF USING HIGH AND LOW-VELOCITY TEST PARTICLES}

Let us consider two test particles - particle A and particle B. Particle A has an extreme recession velocity of $10^{6} \mathrm{~m} \mathrm{~s}^{-1}$, whereas particle B has a recession velocity of just $0.4 \mathrm{~m} \mathrm{~s}^{-1}$.

Initially, particle A begins expanding, after 4 seconds, particle $\mathrm{B}$ begins expanding and is observed for 1 second. By the time particle B is observed for 1 second, particle A has already been expanding for 5 seconds. 
Since particle A began expanding before, therefore, logically, as compared to particle B, particle A will undoubtedly be further away than expected.

The distance covered by particle $\mathrm{A}$ in 5 seconds with a recession velocity of $10^{6} \mathrm{~m} \mathrm{~s}^{-1}$ is $5 \times 10^{6} \mathrm{~m}$, whereas the distance covered by particle $\mathrm{B}$ in 1 second with a recession velocity of $0.4 \mathrm{~m} \mathrm{~s}^{-1}$ is $0.4 \mathrm{~m}$.

The slope or the expansion rate for these particles is obtained by using the relation,

$$
H=\frac{v}{D}
$$

where $H$ is the slope or the expansion rate $\left(\mathrm{m} \mathrm{s}^{-1} \mathrm{~m}^{-1}\right), v$ is the recession velocity of the particles $\left(\mathrm{m} \mathrm{s}^{-1}\right)$, and $D$ is the distance covered by them $(\mathrm{m})$. The inverse of slope or the expansion rate $\left(1 / H\right.$ or $\left.H^{-1}\right)$ gives back the time $\left(t_{H}\right)$ in seconds.

The value of slope or the expansion rate for particle A with a whopping recession velocity of $10^{6} \mathrm{~m} \mathrm{~s}^{-1}$ turns out to be 0.2 $\mathrm{m} \mathrm{s}^{-1} \mathrm{~m}^{-1}$, whereas for particle $\mathrm{B}$, the value of slope or the expansion rate with a mere recession velocity of just $0.4 \mathrm{~m} \mathrm{~s}^{-1}$ turns out to be $1 \mathrm{~m} \mathrm{~s}^{-1} \mathrm{~m}^{-1}$.

The value of slope or the expansion rate for particle A even with an extreme recession velocity of $10^{6} \mathrm{~m} \mathrm{~s}^{-1}$ is much lower ( 5 times lower) than the value of slope or the expansion rate for particle B even with a mere recession velocity of just $0.4 \mathrm{~m} \mathrm{~s}^{-1}$. Does this scientifically imply that particle A with high recession velocity of $10^{6} \mathrm{~m} \mathrm{~s}^{-1}$ is decelerating, whereas particle B with low recession velocity of $0.4 \mathrm{~m} \mathrm{~s}^{-1}$ is accelerating?

$10^{6} \mathrm{~m} \mathrm{~s}^{-1}$ - recession velocity of particle A is $2.5 \times 10^{6}$ times higher than the recession velocity of particle B! Such high recession velocity of particle A does not indicate in any way a low recession velocity, or a slower rate of expansion, or deceleration due to the gravitational attraction of matter!

Then why is particle A with a whopping recession velocity of $10^{6} \mathrm{~m} \mathrm{~s}^{-1}$ yielding a lower value of slope or a slower rate of expansion, thereby suggesting deceleration as compared to particle B with a minuscule recession velocity of just $0.4 \mathrm{~m} \mathrm{~s}^{-1}$ ?

There is absolutely no other reason for an object with high recession velocity to yield a lower value of slope (or a slower rate of expansion, thereby suggesting deceleration) and then be further away than expected, unless it began expanding before.

As already discussed, even with high recession velocity (no matter how high), an object that begins expanding before will never yield a value of slope, or the expansion rate that is higher than the value of slope, or the expansion rate for an object with low recession velocity (no matter how low) that begins expanding comparatively later.

Therefore, we should never compare the slope and thus the expansion rate of such objects, doing so, without any doubt, will result into the apparent transition of Universe's expansion from deceleration to acceleration - an object with high recession velocity that began expanding before will be further away than expected and will appear to be decelerating, whereas an object with low recession velocity that began expanding comparatively later will appear to be accelerating. Requiring mysterious dark energy of unknown origin to explain this apparent transition from deceleration to acceleration would complicate things to an unimaginable extent.

Comparing the slope and thus the expansion rate of highrecession-velocity object that began expanding before, with the slope and thus the expansion rate of low-recession-velocity object that began expanding comparatively later, causes the high-recession-velocity object to appear as if it is receding slower than expected as compared to the low-recession-velocity object.

It is only the result of this comparison that particle A even with an extreme recession velocity of $10^{6} \mathrm{~m} \mathrm{~s}^{-1}$ appears to be expanding at a slower rate (decelerating) as compared to particle B with a mere recession velocity of just $0.4 \mathrm{~m} \mathrm{~s}^{-1}$.

\section{GRAPHICAL CONFIRMATION}

To further confirm the credibility of this undiscovered aspect, it is necessary to plot some graphical relationships for such scenario where an object with high recession velocity (high redshift) begins expanding before, and an object with low recession velocity (low redshift) begins expanding comparatively later. Therefore, we will consider 11 test particles that have been assigned random recession velocities. These test particles expand consecutively (one particle after another). Based on calculations, we will plot some graphical relationships o verify if this undiscovered aspect perfectly mimics cosmic acceleration.

\subsection{Velocity-distance relationship}

Initially, particle A (3517.60 $\left.\mathrm{m} \mathrm{s}^{-1}\right)$ begins expanding, 0.1 second later, particle B $\left(2983.93 \mathrm{~m} \mathrm{~s}^{-1}\right)$ begins expanding, the expansion of particle $\mathrm{B}$ is followed by the expansion of particle C $\left(2648.64 \mathrm{~m} \mathrm{~s}^{-1}\right)$ after another 0.1 second. Consecutive expansion of particles continues in the same way for particle D $\left(2496.43 \mathrm{~m} \mathrm{~s}^{-1}\right)$, particle E $\left(2223.52 \mathrm{~m} \mathrm{~s}^{-1}\right)$, particle F $(1676.20$ $\mathrm{m} \mathrm{s}^{-1}$ ), particle $\mathrm{G}\left(1219.96 \mathrm{~m} \mathrm{~s}^{-1}\right)$, particle $\mathrm{H}\left(917.97 \mathrm{~m} \mathrm{~s}^{-1}\right)$, and particle I $\left(768.62 \mathrm{~m} \mathrm{~s}^{-1}\right)$. Particle $\mathrm{J}\left(530.48 \mathrm{~m} \mathrm{~s}^{-1}\right)$ and particle $\mathrm{K}$ $\left(257.85 \mathrm{~m} \mathrm{~s}^{-1}\right)$ are the last particles to expand, and they expand at the same time and are observed for 1 second. By the time these last two particles expand and are observed for 1 second, particle A has already been expanding for 1.9 second, and particle B for 1.8 second, this becomes their respective observation time.

The velocity-distance relationship for these 11 test particles has been plotted in Figure 5. The plot is remarkably similar to the redshift-distance relationship for 588 type Ia supernovae (Figure 1), and to the velocity-distance relationship for 5 type Ia supernovae (Figure 17). The deviation from linearity in Figure 5 clearly indicates that remote particles are not only further away than expected, but they are also yielding a lower value of slope, or a slower rate of expansion (deceleration) even with high recession velocities as compared to the local particles that are yielding a higher value of slope, or a faster rate of expansion (acceleration) even with low recession velocities - apparent transition from deceleration to acceleration (past to present).

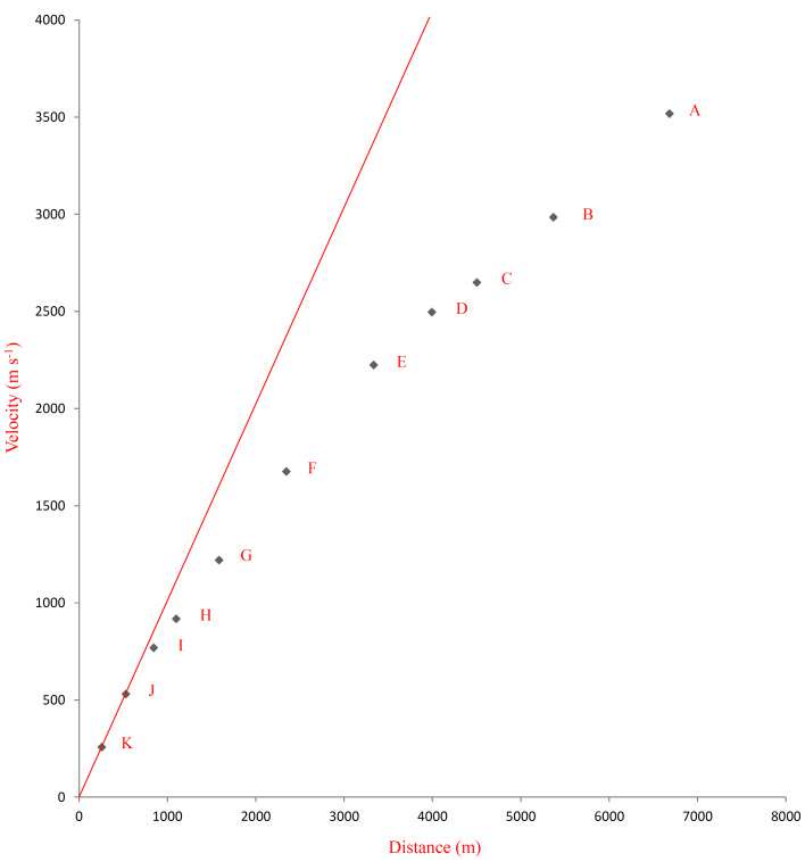

Figure 5. Velocity-distance relationship for 11 test particles (local and remote particles) expanding consecutively (one particle after another). Distances to remote particles are larger than expected with respect to local particles without acceleration. In other words, expansion initiated for remote particles before it did for local particles (see Figure 17 for comparison)

The value of slope for the most distant remote particle A in Figure 5 is $0.526315789 \mathrm{~m} \mathrm{~s}^{-1} \mathrm{~m}^{-1}$ (a lower value of slope, or a slower rate of expansion even with high recession velocity of $3517.60 \mathrm{~m} \mathrm{~s}^{-1}$ ) - does this imply deceleration? The inverse of this slope gives the original observation/expansion time of 1.9 second.

For local particles, particle $\mathrm{J}$ and particle $\mathrm{K}$, the value of slope (slope of the red line) turns out to be $1 \mathrm{~m} \mathrm{~s}^{-1} \mathrm{~m}^{-1}$ (a higher value of slope, or a faster rate of expansion even with low recession velocities of $530.48 \mathrm{~m} \mathrm{~s}^{-1}$ and $257.85 \mathrm{~m} \mathrm{~s}^{-1}$ respectively) - does this imply acceleration? The inverse of this slope gives the original observation/expansion time of 1 second.

The recession velocity of particle $\mathrm{A}$ is 6.63 times higher than the recession velocity of particle $\mathrm{J}$, and 13.64 times higher than the recession velocity of particle K. Particle A still happens to yield a lower value of slope, thereby suggesting a slower rate of expansion or deceleration as compared to these two particles (not to mention again that particle $\mathrm{A}$ is further away than expected as compared to these two particles). 
Could there be any other scientifically-valid reason why an object with high recession velocity would be yielding a lower value of slope, thereby suggesting a slower rate of expansion or deceleration and then be further away than expected as compared to an object with low recession velocity?

There is absolutely no other reason for an object with high recession velocity to yield a lower value of slope (or a slower rate of expansion, thereby suggesting deceleration) and then be further away than expected, unless it began expanding before.

High recession velocities of remote objects yielding a lower value of slope do not indicate their deceleration. Similarly, low recession velocities of local objects yielding a higher value of slope do not indicate their acceleration. Requiring mysterious dark energy of unknown origin to explain such transition would unnecessarily complicate things to an unimaginable extent.

Since expansion began for remote particles before it did for local particles, therefore, remote particles are not only further away than expected, but they are also yielding a lower value of slope (or a slower rate of expansion) even with high recession velocities as compared to the higher value of slope (or a faster rate of expansion) for local particles even with low recession velocities. It therefore appears that local particles are expanding at a faster rate as compared to remote particles. One would therefore be forced into believing that local particles, as compared to remote particles, are accelerating.

\subsection{Expansion rate versus time relationship}

Although the observational fact that a remote object which happens to be further away than expected yields a slower rate of expansion even with high recession velocity as compared to a local object that yields a faster rate of expansion even with low recession velocity is the most compelling evidence to suggest that remote structures began expanding before the expansion got initiated for local structures, however, to further confirm upon this aspect, we will plot expansion rate versus time relationship for such scenario where remote particles with high recession velocities (high redshifts) began expanding before the expansion got initiated for local particles with low recession velocities (low redshifts).

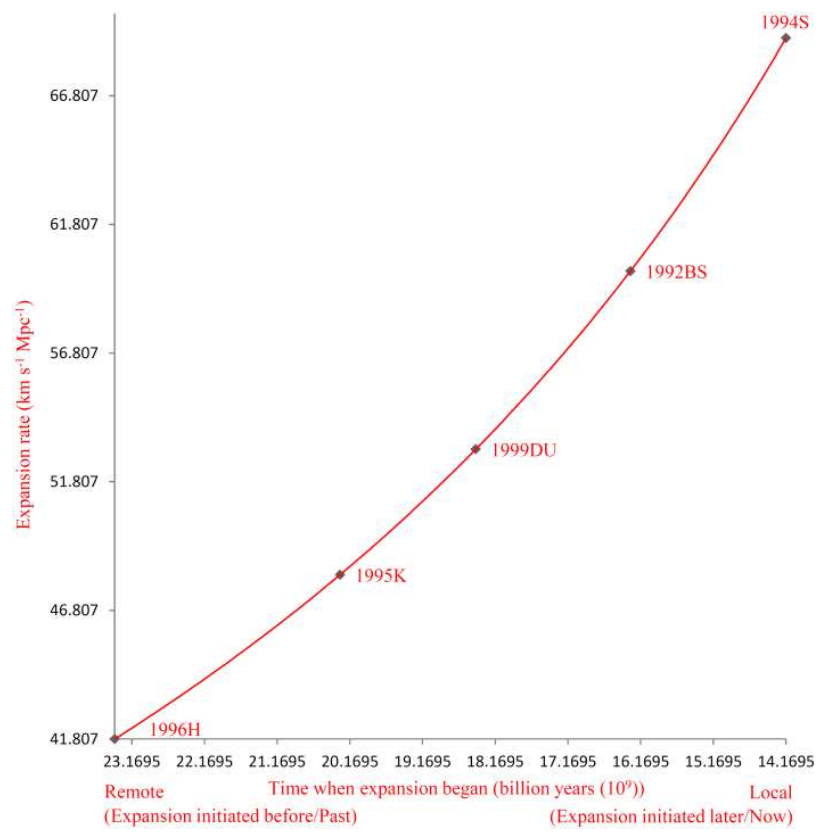

Figure 6. Plot of expansion rate versus time when expansion began (measured from past to present) for 5 type Ia supernovae (remote and local supernovae from Figure 1) shows an accelerating expansion (expansion rate increasing with time). Expansion rate for remote supernovae that are further away than expected (see Figure 1 and Figure 17) is lower even with high recession velocities as compared to the expansion rate for nearby local supernovae even with low recession velocities.

Here in Figure 6, we see that expansion rate is increasing with time; expansion rate for remote supernovae is lower than the expansion rate for local supernovae - Universe is expanding slower in the past and is expanding faster now.

Now we need to plot the expansion rate versus time relationship when particles with high recession velocities (high redshifts) began expanding before the expansion got initiated for particles with low recession velocities (low redshifts).
As discussed previously in Section 6.1, initially, the highrecession-velocity (high-redshift) particle A began expanding, 0.1 second later, particle $\mathrm{B}$ began expanding, expansion of particle B was followed by the expansion of particle C after another 0.1 second. Consecutive expansion of particles continued in the same way for remaining particles. Lowrecession-velocity (low-redshift) particles - particle $\mathbf{J}$ and particle $\mathrm{K}$ were the last particles to expand, and, they expanded at the same time and were observed for 1 second. By the time these last two particles expanded and were observed for 1 second, particle A had already been expanding for 1.9 second, particle B for 1.8 second, particle C for 1.7 second, and so on.

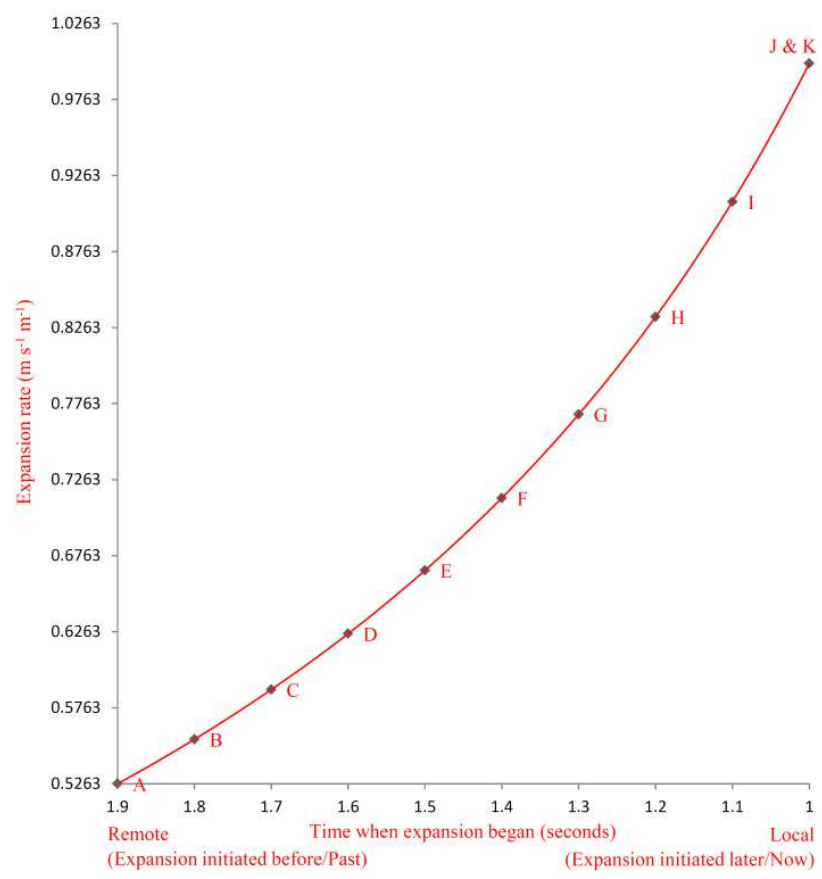

Figure 7. Plot of expansion rate versus time when expansion began (measured from past to present) for 11 test particles (remote and local particles from Figure 5) mimics an accelerating expansion (expansion rate appears to be increasing with time) when remote particles with high recession velocities began expanding before the expansion got initiated for local particles with low recession velocities. Expansion rate for remote particles that are further away than expected (see Figure 5) is lower even with high recession velocities as compared to the expansion rate for nearby local particles even with low recession velocities, similar to what we observe for supernovae in Figure 6.

Here in Figure 6 and Figure 7, the time when expansion began has been obtained by using the relation,

$$
t_{H}=\frac{1}{H}
$$

See $H$ from equation (1). The similarities encountered while plotting Figure 6 and Figure 7 (expansion rate increasing with time) are strong enough to indicate that remote structures with high recession velocities (high redshifts) were the ones that began expanding before the expansion got initiated for local structures with low recession velocities (low redshifts).

\subsection{Expansion factor versus time relationship}

If redshift helps indicating the size of the Universe now as compared to its size when the light was emitted, then the study conducted here based on redshifts of 10 test particles should also help us confirm that remote structures with high redshifts began expanding before the expansion got initiated for local structures with low redshifts.

Velocity of particles $(v)$ in $\mathrm{m} \mathrm{s}^{-1}$ has been converted to redshift (z) by using the relation,

$$
z=\frac{v}{c}
$$

where $c$ is the velocity of light in $\mathrm{m} \mathrm{s}^{-1}$. Similarly, the lighttravel-time $\left(t_{c}\right)$ in seconds corresponding to the distance $(D)$ to particles in meters has been calculated by using the relation,

$$
t_{c}=\frac{D}{c}
$$

Just like a high-redshift remote supernova that we observe to be further away than expected as a result of cosmic acceleration (Figure 1 and Figure 2), we are observing the high-redshift 


\section{Karan R.Takkhi}

remote particle A which is also further away than expected (however, not because of acceleration, but because it began expanding before as per consecutive expansion (Figure 5)), at a distance $(D)$ of $2.227813333 \times 10^{-5}$ light seconds $(6683.44 \mathrm{~m})$ with a redshift $(z)$ of $1.172533333 \times 10^{-5}\left(3517.60 \mathrm{~m} \mathrm{~s}^{-1}\right)$.

The percentage of shift in the spectral lines towards the red-end of the electromagnetic spectrum for particle $\mathrm{A}$ is $1.172533333 \times 10^{-3} \%$ (this also corresponds to the percentage of expansion that has occurred while the light from particle A has been in transit before reaching us), in other words, the Universe is $1.172533333 \times 10^{-3} \%$ larger now than it was when the light was emitted.

To get a factor (expansion factor) which would help us calculate the time when the Universe was $100 \%$ smaller than now, we need to divide $100 \%$ by the percentage of shift in the spectral lines towards the red-end of the electromagnetic spectrum for a particular particle (this percentage of shift in the spectral lines also corresponds to the percentage of expansion that has occurred while the light from that particular particle has been in transit before reaching us), therefore, the expansion factor for the remote particle $\mathrm{A}$ is,

$$
\frac{100}{1.172533333 \times 10^{-3}}=85285.4219
$$

(The expansion factor can also be obtained directly by taking an inverse of the redshift $(z))$. This factor suggests that we will have to reverse the expansion 85285.4219 times back into the past when the scale factor was zero and everything was at the same place - the Big Bang (since $t=D / v$, and $v=c z$, therefore, $t=D / c z$, on isolating we get, $D / c$ which is the lighttravel-time $\left(t_{\mathrm{c}}\right)$, and $1 / z$ which is the expansion factor, moreover, $\left.z=t_{\mathrm{c}} / t\right)$. Therefore, multiplying the expansion factor obtained for particle A (85285.4219) with its light-travel-time in seconds $\left(2.227813333 \times 10^{-5}\right)$ gives back the original expansion time $(t)$ for particle A (1.9 second), that is, the time $(t)$ in the past when particle A began expanding (expansion of particles has occurred at a steady rate; we have not subjected any test particle to acceleration or deceleration).

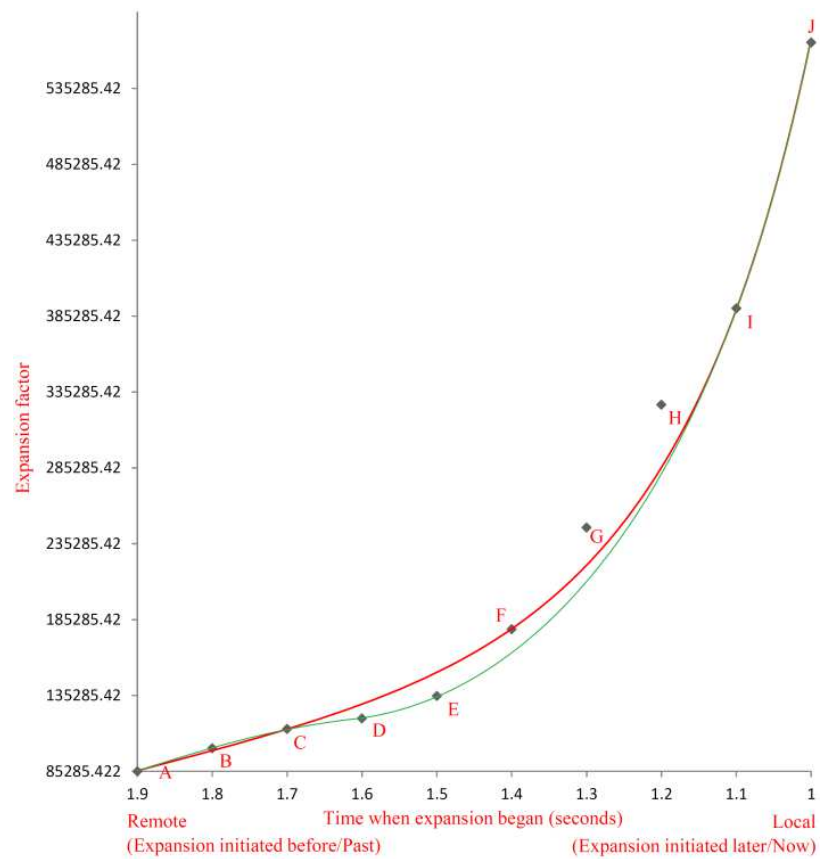

Figure 8. Plot of expansion factor versus time when expansion began (measured from past to present) for 10 test particles (remote and local particles from Figure 5) mimics an accelerating expansion (expansion factor increasing exponentially with time - red curve) when remote particles with high redshifts began expanding before the expansion got initiated for local particles with low redshifts. The green curve (overlapping the red curve) traces out an expansion that initially decelerates before accelerating. (Also see Figure 14).

Here in Figure 8, the time when expansion began for test particles has been obtained by multiplying their expansion factor with their light-travel-time in seconds, and, this is consistent with time when expansion began for these test particles obtained in Figure 7 by using equation (2).

It can be seen that high-redshift remote particles that began expanding before yield a smaller expansion factor (expansion factor for remote particle A (85285.4219)) as compared to the expansion factor that increases exponentially with time for lowredshift local particles that began expanding comparatively later (expansion factor for local particle J (565525.5618)).

We will now follow the same method for type Ia supernovae (remote and local supernovae from Figure 1) to see if they also exhibit a similar expansion factor versus time relationship. A similar relationship will further help us confirm that remote structures with high redshifts began expanding before the expansion got initiated for local structures with low redshifts.

We have remote supernova $1996 \mathrm{H}$ at a distance $(D)$ of 14.5043 Gly with a redshift $(z)$ of 0.62 ; this remote supernova is further away than expected as compared to local supernovae. The percentage of shift in the spectral lines towards the red-end of the electromagnetic spectrum for this supernova is $62 \%$ (this also corresponds to the percentage of expansion that has occurred while the light from this remote supernova has been in transit before reaching us), in other words, the Universe is $62 \%$ larger now than it was when the light was emitted.

To get the expansion factor which would help us calculate the time when the Universe was $100 \%$ smaller than now, we need to divide $100 \%$ by the percentage of shift in the spectral lines towards the red-end of the electromagnetic spectrum for a particular supernova (this percentage of shift in the spectral lines also corresponds to the percentage of expansion that has occurred while the light from that particular supernova has been in transit before reaching us), therefore, the expansion factor for the remote supernova $1996 \mathrm{H}$ is,

$$
\frac{100}{62}=1.612903226
$$

This factor suggests that we will have to reverse the expansion 1.612903226 times back into the past when the scale factor was zero and everything was at the same place - the Big Bang. Therefore, multiplying the expansion factor obtained for supernova $1996 \mathrm{H}(1.612903226)$ with its light-travel-time in years $\left(14.510739 \times 10^{9}\right.$ years $)$ gives back the original expansion time $(t)$ for supernova $1996 \mathrm{H}\left(23.4044 \times 10^{9}\right.$ years $)$, that is, the time $(t)$ in the past when supernova $1996 \mathrm{H}$ began expanding.

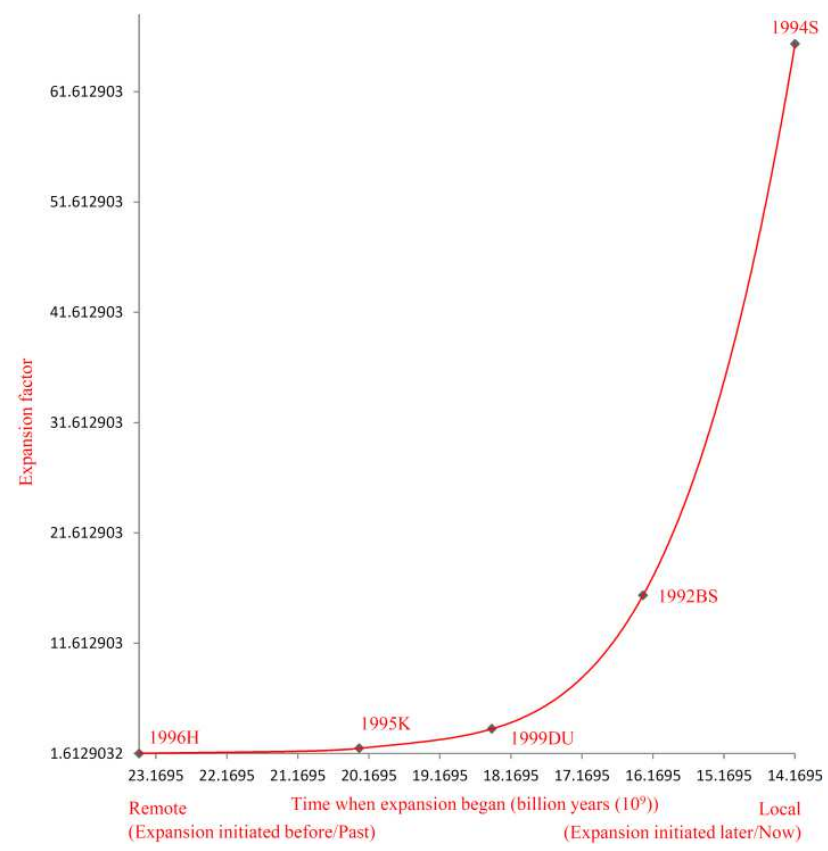

Figure 9. Plot of expansion factor versus time when expansion began (measured from past to present) for 5 type Ia supernovae (remote and local supernovae from Figure 1) shows an accelerating expansion (expansion factor increasing exponentially with time).

Here in Figure 9, the time when expansion began for supernovae has been obtained by multiplying their expansion factor with their light-travel-time in years, and, this is consistent with time when expansion began for these supernovae obtained in Figure 6 by using equation (2).

It can be seen that high-redshift remote supernovae that are further away than expected as a result of cosmic acceleration are yielding a smaller expansion factor (expansion factor for remote supernova $1996 \mathrm{H}$ (1.612903226)) as compared to the expansion factor that is increasing exponentially with time for low-redshift local supernovae (expansion factor for local supernova 1994S (65.93696426)). 
In Figure 8, high-redshift remote particles that are further away than expected are yielding a smaller expansion factor as compared to the expansion factor that increases exponentially with time for low-redshift local particles, and, such exponential increase in expansion factor has occurred when remote particles with high redshifts began expanding before the expansion got initiated for local particles with low redshifts, in other words, such exponential increase in expansion factor has occurred without subjecting any test particle to acceleration or deceleration.

In Figure 9, high-redshift remote supernovae that are further away than expected are also yielding a smaller expansion factor as compared to the expansion factor that increases exponentially with time for low-redshift local supernovae - similar to what we observe in Figure 8 using test particles.

Such similarity further confirms that remote structures with high redshifts began expanding before the expansion got initiated for local structures with low redshifts, for this reason, remote supernovae are further away than expected as compared to the nearby local supernovae.

\subsection{Expansion factor versus light-travel-time relationship}

In the previous section we obtained the expansion factor, the light-travel-time, and the time when expansion began. We plotted expansion factor versus time (time when expansion began) relationship and found expansion factor increasing exponentially with time (past to present - remote to local) when remote particles with high redshifts began expanding before the expansion got initiated for local particles with low redshifts (as per consecutive expansion), a similar relationship was also obtained for 5 type Ia supernovae. The time when expansion began was obtained by multiplying the expansion factor with the light-travel-time (also consistent with equation (2)).

Here we will consider plotting expansion factor versus lighttravel-time relationship for such scenario when remote particles with high redshifts began expanding before the expansion got initiated for local particles with low redshifts, we will then plot expansion factor versus light-travel-time relationship for 5 type Ia supernovae to see if they also exhibit a similar relationship. A similar relationship, if obtained, will further help us confirm that remote structures with high redshifts were the ones that began expanding before the expansion got initiated for local structures with low redshifts.

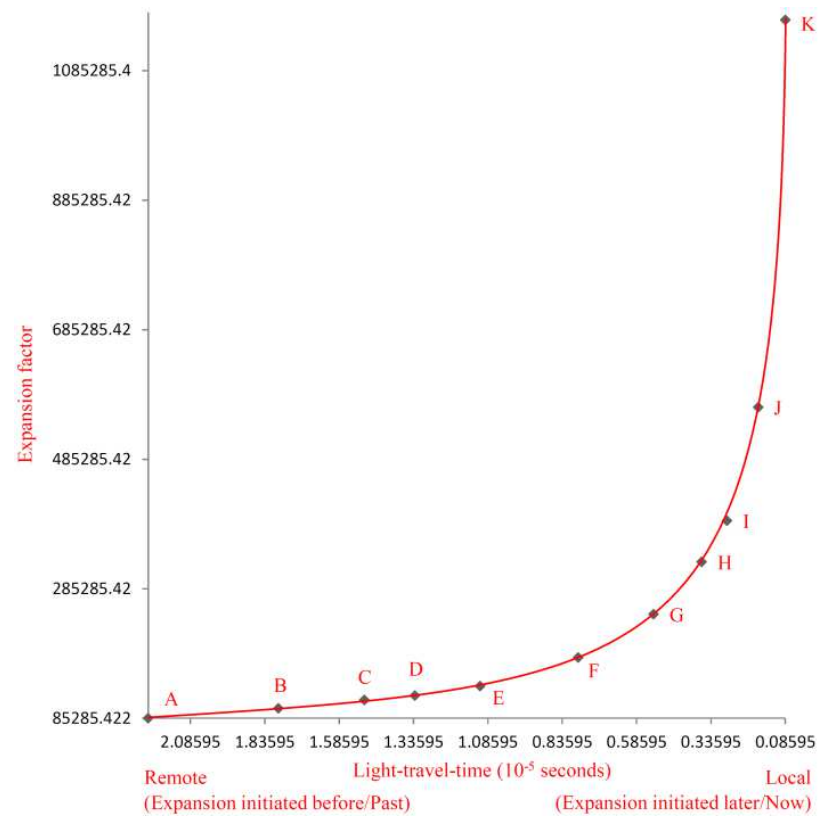

Figure 10. Plot of expansion factor versus light-travel-time (measured from past to present) for 11 test particles (remote and local particles from Figure 5) mimics an accelerating expansion (expansion factor increasing exponentially with time) when remote particles with high redshifts began expanding before the expansion got initiated for local particles with low redshifts. Figure is consistent with Universe that is accelerating as expansion factor is increasing exponentially with time.

Plot of expansion factor versus light-travel-time relationship (measured from past to present) for 5 type Ia supernovae (remote and local supernovae from Figure 1) shows a similar relationship in Figure 11 as obtained here in Figure 10 for 11 test particles that expanded consecutively - expansion factor increasing exponentially with time.

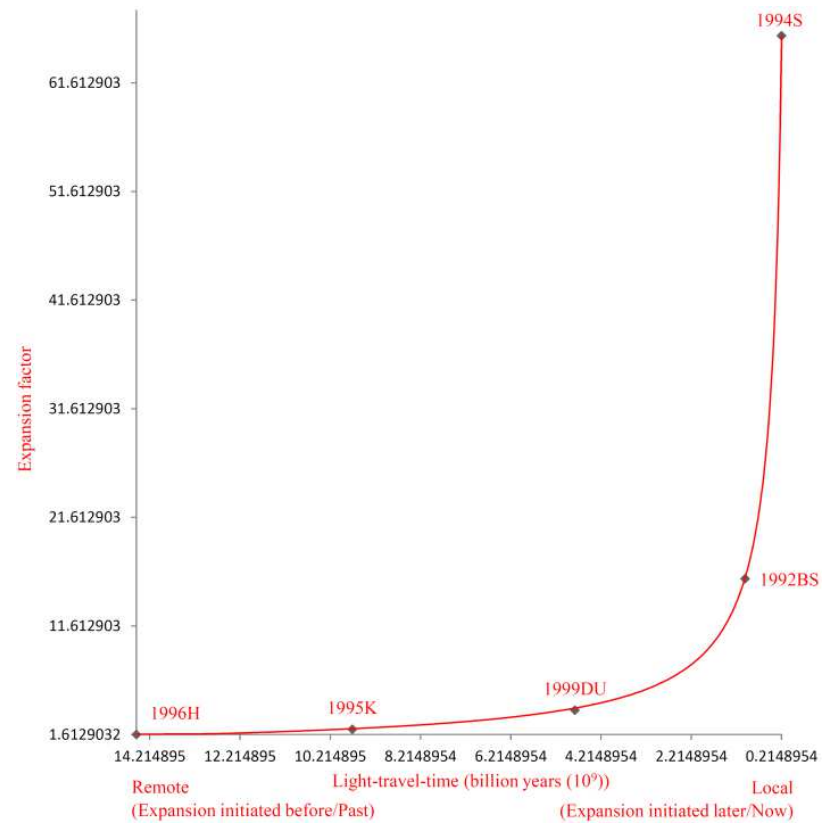

Figure 11. Plot of expansion factor versus light-travel-time (measured from past to present) for 5 type Ia supernovae (remote and local supernovae from Figure 1) shows an accelerating expansion (expansion factor increasing exponentially with time).

\subsection{Scale factor versus light-travel-time relationship}

Evolution of Universe's scale factor with time plays an important role in determining the type of Universe we live in. Measuring the variation of Universe's scale factor with time (past to present - remote to local) would not only help us determine the type of Universe we live in, but it would also help us predict its ultimate fate.

The study conducted here based on redshifts of 11 test particles should also help us confirm that remote structures with high redshifts began expanding before the expansion got initiated for local structures with low redshifts.

We have remote supernova $1996 \mathrm{H}$ at a distance $(D)$ of 14.5043 Gly with a redshift $(z)$ of 0.62 . As a result of cosmic acceleration, this remote supernova is further away than expected as compared to the nearby local supernovae. The scale factor $(a(t))$ which denotes the size of the Universe when the light was emitted is obtained by using the relation,

$$
a(t)=\frac{1}{1+z}
$$

where $z$ is the redshift. The light-travel-time $\left(t_{c}\right)$ in years corresponding to the distance $(D)$ to the supernovae in meters has been calculated by using equation (4).

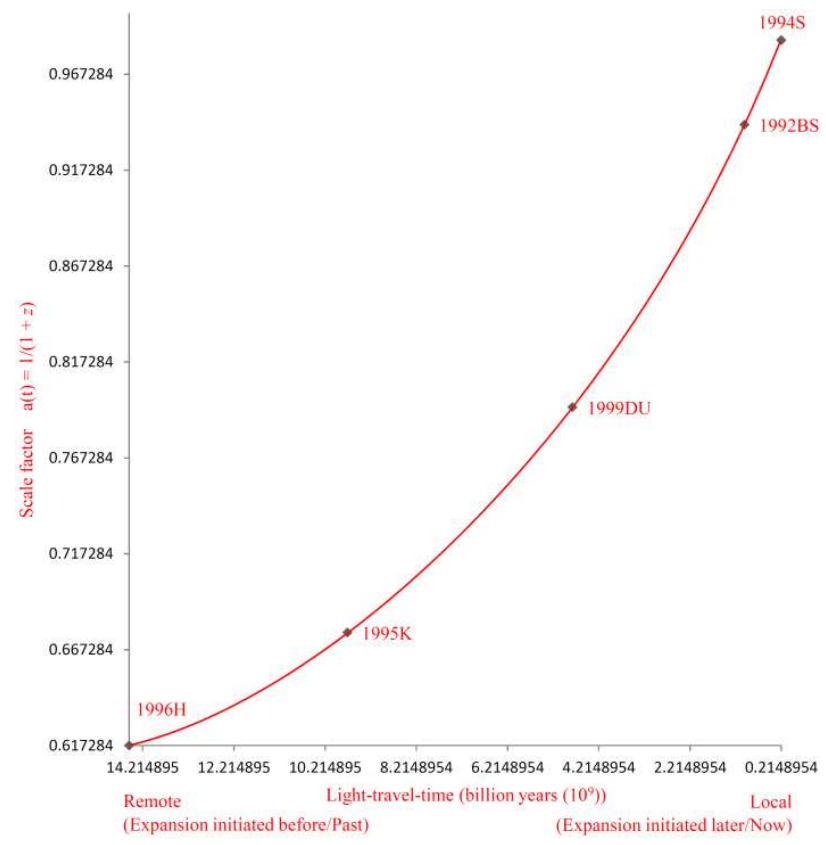

Figure 12. Plot of scale factor versus light-travel-time (measured from past to present) for 5 type Ia supernovae (remote and local supernovae from Figure 1) shows an accelerating expansion (scale factor increasing with time). 
Here in Figure 12, high-redshift remote supernovae that are further away than expected are yielding a smaller scale factor (scale factor for remote supernova $1996 \mathrm{H}(0.61728395)$ ) as compared to the scale factor that increases with time for lowredshift local supernovae (scale factor for local supernova 1994S (0.985060571)), in fact, scale factor evolution with time (past to present) exhibiting a curve as obtained in Figure 12 clearly depicts an accelerating expansion.

Now we need to plot the scale factor versus light-travel-time relationship when remote particles with high redshifts began expanding before the expansion got initiated for local particles with low redshifts as per consecutive expansion.

Velocity $(v)$ of particles in $\mathrm{m} \mathrm{s}^{-1}$ has been converted to redshift (z) by using equation (3). Similarly, the light-travel-time $\left(t_{c}\right)$ in seconds corresponding to the distance $(D)$ to the particles in meters has been calculated by using equation (4).

Just like a high-redshift remote supernova that we observe to be further away than expected (as a result of cosmic acceleration) as shown in Figure 1 and Figure 2, we are observing the high-redshift remote particle A which is also further away than expected (however, not because of acceleration, but because it began expanding before as a result of consecutive expansion (Figure 5)) at a distance $(D)$ of $2.227813333 \times 10^{-5}$ light seconds (6683.44 m), exhibiting a redshift $(z)$ of $1.172533333 \times 10^{-5}\left(3517.60 \mathrm{~m} \mathrm{~s}^{-1}\right)$. The scale factor $(a(t))$ for these 11 test particles has been obtained by using equation (7).

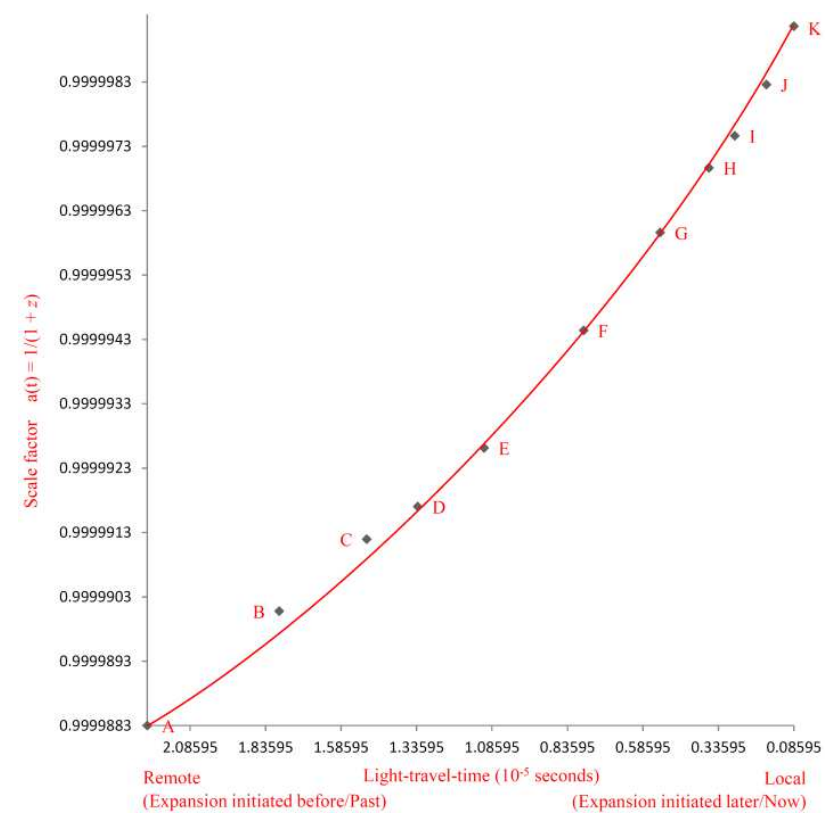

Figure 13. Plot of scale factor versus light-travel-time (measured from past to present) for 9 test particles (remote and local particles from Figure 5) mimics an expansion that accelerates (scale factor increasing with time) when remote particles with high redshifts began expanding before the expansion got initiated for local particles with low redshifts. Figure is consistent with Universe that is accelerating as scale factor is increasing with time (in agreement with Figure 12).

As shown above in Figure 13, scale factor versus light-traveltime relationship for 9 test particles is consistent with an expansion that is accelerating with time (past to present) even when the test particles have not been subjected to acceleration, whereas in Figure 14, scale factor versus light-travel-time relationship for 11 test particles is consistent with an expansion that first decelerates, and then accelerates even when the test particles have not been subjected to deceleration or acceleration. This apparent transition of particles' expansion from deceleration to acceleration as obtained here in Figure 14 can also be seen in Figure 8 as traced by the green curve.

It is evidently clear from Figure 13 and Figure 14 that highredshift remote particles that are further away than expected are yielding a smaller scale factor (scale factor for remote particle A (0.999988274)) as compared to the scale factor that increases with time for low-redshift local particles (scale factor for local particle K (0.99999914)). Why should test particles that were not at all subjected to deceleration or acceleration exhibit scale factor evolution consistent with a Universe whose expansion is accelerating over time?

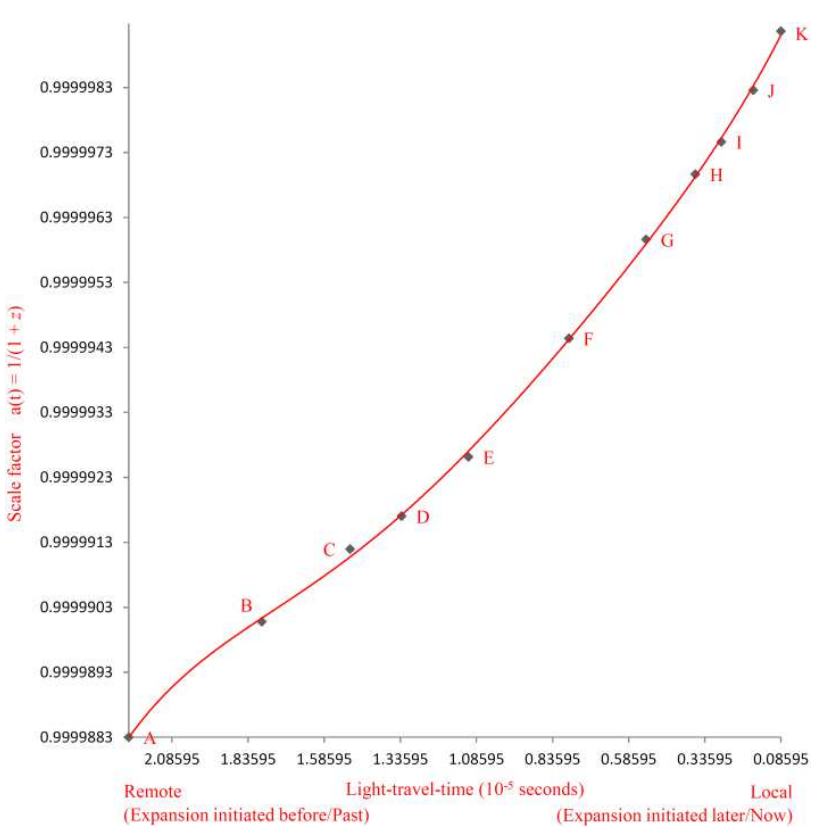

Figure 14. Plot of scale factor versus light-travel-time (measured from past to present) for 11 test particles (remote and local particles from Figure 5) mimics an expansion that first decelerates, and then accelerates when remote particles with high redshifts began expanding before the expansion got initiated for local particles with low redshifts. Figure is consistent with Universe that first decelerates, and then accelerates (in agreement with Figure 15).

In Figure 13 and Figure 14, the observed increase in scale factor with time (past to present) for test particles (remote to local) has occurred when remote particles with high redshifts began expanding before the expansion got initiated for local particles with low redshifts as per consecutive expansion, in other words, such increase in scale factor has occurred without subjecting any test particle to deceleration or acceleration.

In Figure 12 and Figure 15, far and near supernovae are also exhibiting a similar scale factor evolution as measured from past to present - high-redshift remote supernovae that are further away than expected are also yielding a smaller scale factor as compared to the scale factor that increases with time for low-redshift local supernovae - similar to what we observe here in Figure 13 and Figure 14 using test particles.

Such similarity further confirms that remote structures with high redshifts were the ones that began expanding before the expansion got initiated for local structures with low redshifts in agreement with consecutive expansion epochs of the Universe that preceded the current expansion epoch. Remote supernovae are therefore further away than expected as compared to the nearby local supernovae.

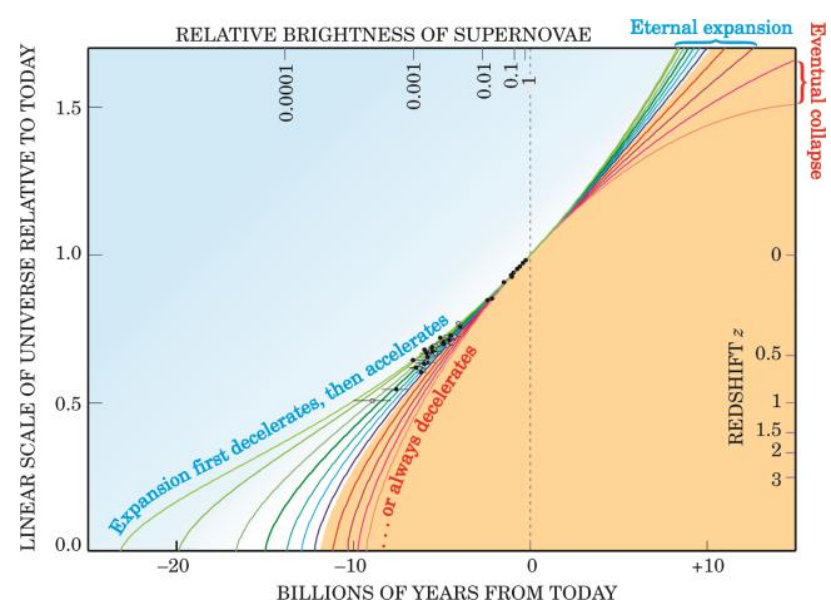

Figure 15. Plot of scale factor versus light-travel-time (measured from past to present) for type Ia supernovae helps depicting the expansion history of the Universe - the plot depicts Universe's expansion that first decelerates, and then accelerates. High-redshift remote supernovae that are further away than expected are yielding a smaller scale factor as compared to the scale factor that is increasing with time for the nearby local supernovae with low redshifts, thereby indicating accelerating expansion of the Universe. Credit: Perlmutter S., Physics Today, vol. 56 , 53, page 57, year 2003, reproduced with the permission of the American Institute of Physics, Copyright (2003) American Institute of Physics. https://doi.org/10.1063/1.1580050 
Shedding Light on an Undiscovered Aspect that Perfectly Mimics Cosmic Acceleration 9

6.6. Redshift-distance and velocity-distance relationships

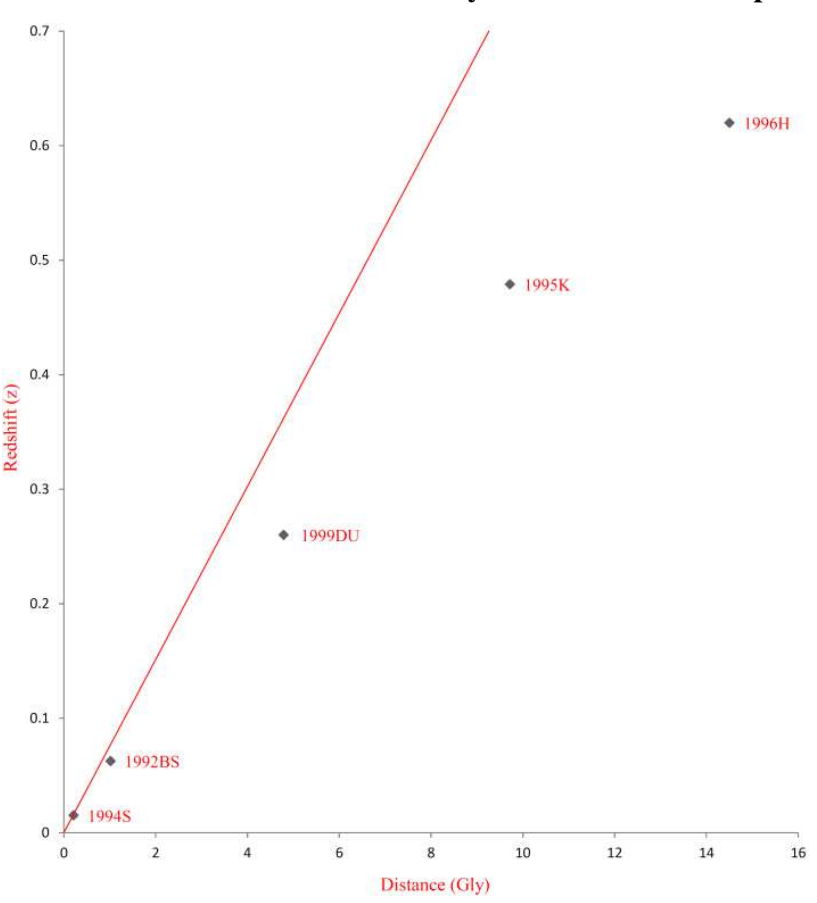

Figure 16. Redshift-distance relationship for 5 type Ia supernovae (local and remote supernovae from Figure 1). Remote supernovae are further away than expected as compared to the nearby local supernovae.

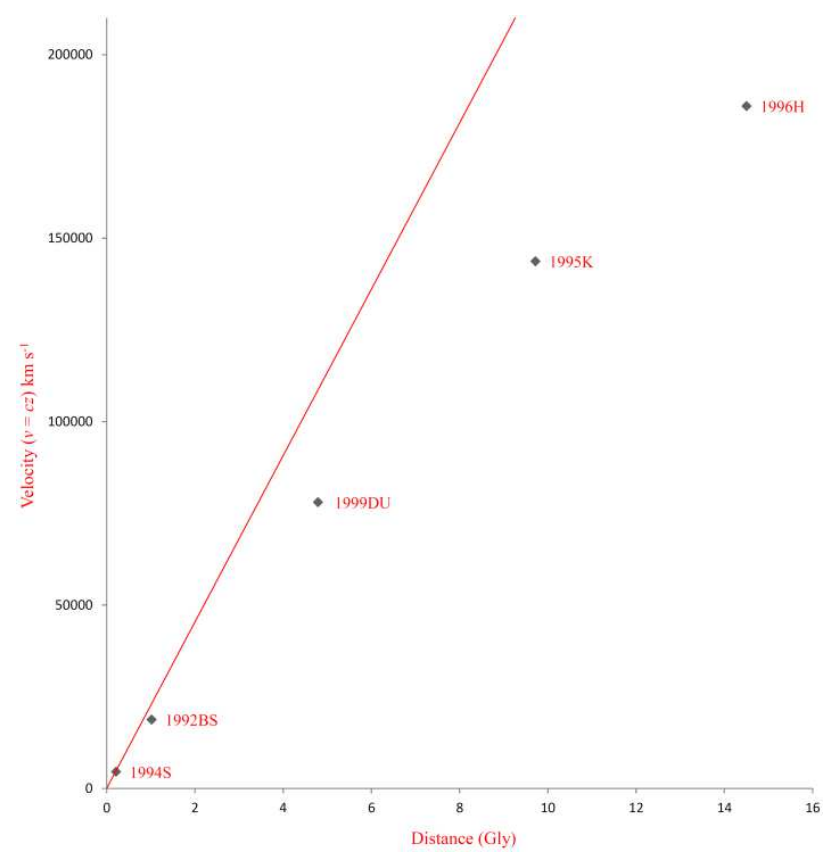

Figure 17. Velocity-distance relationship for 5 type Ia supernovae (local and remote supernovae from Figure 1). Remote supernovae are further away than expected as compared to the nearby local supernovae.

Here we have plotted redshift-distance relationship and velocity-distance relationship for 5 type Ia supernovae from Figure 1. Both these plots, just like Figure 1, demonstrate that the very distant, far away remote supernovae are further away than expected, thereby getting rendered $10 \%$ to $25 \%$ dimmer as compared to their nearby counterparts. This further-away-thanexpected placement of remote supernovae is attributed to an energy component responsible for accelerating the Universe's expansion - without this energy component there would have not been cosmic acceleration, and without cosmic acceleration, remote supernovae would have not been placed further away than expected.

In particular, the velocity-distance relationship for local and remote supernovae (Figure 17) is characteristically similar to the velocity-distance relationship obtained for local and remote test particles (Figure 5), that is, the expansion rate for remote supernovae (Figure 6) is lower even with high recession velocities as compared to the expansion rate for nearby local supernovae even with low recession velocities - this attribute is similar to what we have observed for test particles (Figure 7) without having subjected any of those test particles to deceleration or acceleration. Such similar attributes favour consecutive expansion epochs of the Universe that preceded the current expansion epoch, rather than recent accelerated expansion and a previous decelerated expansion.

\subsection{Distance-redshift relationship}

Redshift-distance relationship already plotted for local and remote supernovae (Figure 1 and Figure 16) shows the deviation from linearity at high redshifts as remote supernovae are further away than expected.

Here we will consider plotting distance-redshift relationship for 5 type Ia supernovae from Figure 1 (these are the same 5 type Ia supernovae that have been considered so far) we will then plot the same relationship for 11 test particles from Figure 5. A similar relationship will further help us confirm that remote structures with high redshifts were the ones that began expanding before the expansion got initiated for local structures with low redshifts - in agreement with consecutive expansion epochs of the Universe that preceded the current expansion epoch.

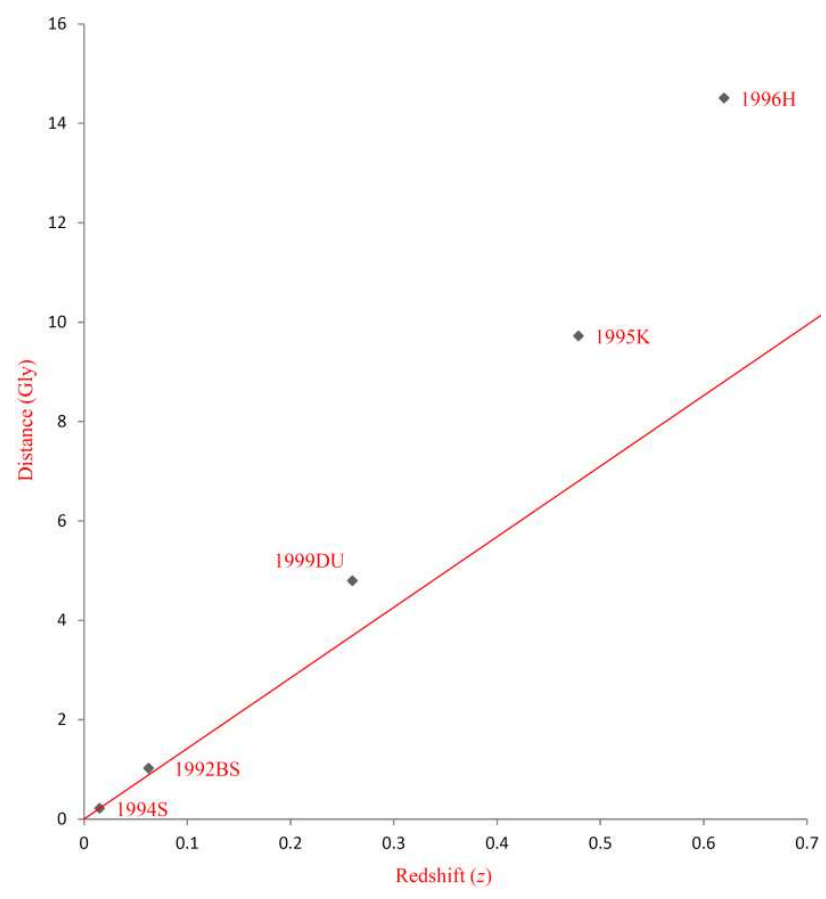

Figure 18. Distance-redshift relationship for 5 type Ia supernovae (local and remote supernovae from Figure 1. High-redshift remote supernovae lie above the line; the deviation from linearity makes it clear enough that the distances to remote supernovae are larger than expected, thereby making them appear $10 \%$ to $25 \%$ dimmer as compared to the nearby local supernovae. It is believed that there is an energy component that has accelerated the expansion of the Universe, thereby placing remote supernovae further away than expected.

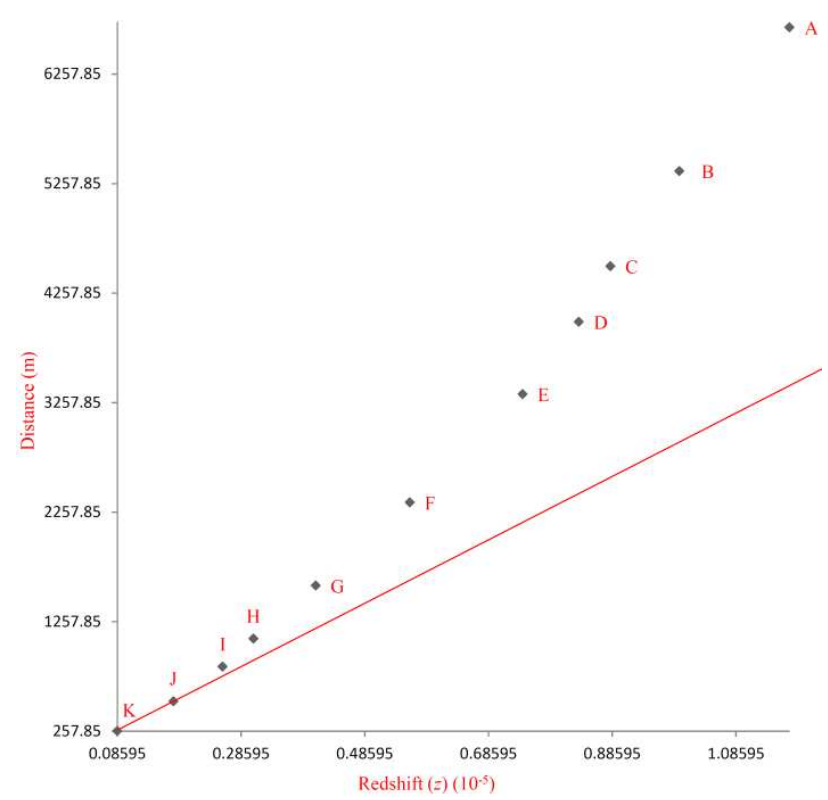

Figure 19. Distance-redshift relationship for 11 test particles (local and remote particles from Figure 5). High-redshift remote particles lie above the line; the deviation from linearity also makes it clear enough that the distances to remote particles are larger than expected. 
The deviation of distance-redshift relationship from linearity at large distances (at high redshifts) depends on how the expansion rate has changed over time. Since deviation from linearity implies distances to type Ia supernovae to be larger than expected and a change in the expansion rate, therefore, it is believed that such feat can only be achieved if the Universe expanded slowly in the past than it does today - expansion of the Universe has accelerated over time (past to present - remote to local) due to an energy component.

Here in Figure 19, there should also be an energy component that has accelerated the expansion of particles, thereby placing remote particles further away than expected. However, as per Section 6.1, we are well aware that not even a single test particle was subjected to deceleration or acceleration, therefore, the presence of an energy component responsible for placing remote particles further away than expected is completely out of question. It is only because of consecutive expansion of particles wherein expansion of one particle is followed by the next in a consecutive manner that we are getting a plot that is characteristically similar to the distance-redshift relationship for 5 type Ia supernovae as shown in Figure 18.

\subsection{Distance modulus versus redshift relationship}

The distance modulus $(\mu)$ is the difference between the apparent magnitude $(m)$ and the absolute magnitude $(M)$, it denotes distances on a logarithmic scale and is given as,

$$
\mu=m-M=5 \log (d)-5
$$

where $d$ is the luminosity distance in parsecs. If we know the distance $(d)$, for instance, we already know the distances to 11 test particles, therefore, we can calculate and obtain the distance modulus $(\mu)$ for them by using the relation,

$$
\mu=5 \log (d)-5
$$

or, by using the relation,

$$
\mu=5 \log \left(\frac{d}{10}\right)
$$

If distance modulus $(\mu)$ is known, we can obtain the distance $(d)$ in parsecs by using the relation,

$$
d=10^{\frac{\mu}{5}+1}
$$

Here in Figure 20, we have plotted distance modulus versus redshift relationship for 5 type Ia supernovae from Figure 1.

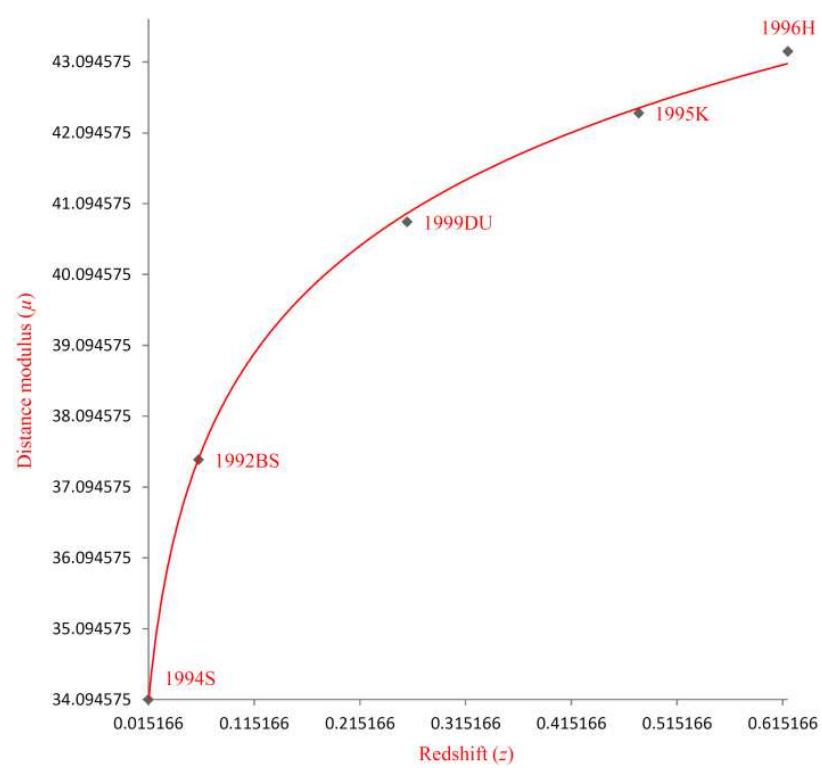

Figure 20. Distance modulus versus redshift relationship for 5 type Ia supernovae (local and remote supernovae from Figure 1). Type Ia supernovae are further away than expected, this confirms the presence of an unknown energy component responsible for accelerating the Universe's expansion, thereby placing remote supernovae further away than expected.

Distance modulus versus redshift relationship for 11 test particles (local and remote particles from Figure 5) has also been plotted in Figure 21. The similar relationship obtained for test particles further helps us confirm that remote structures with high redshifts began expanding before the expansion got initiated for local structures with low redshifts - consistent with consecutive expansion.

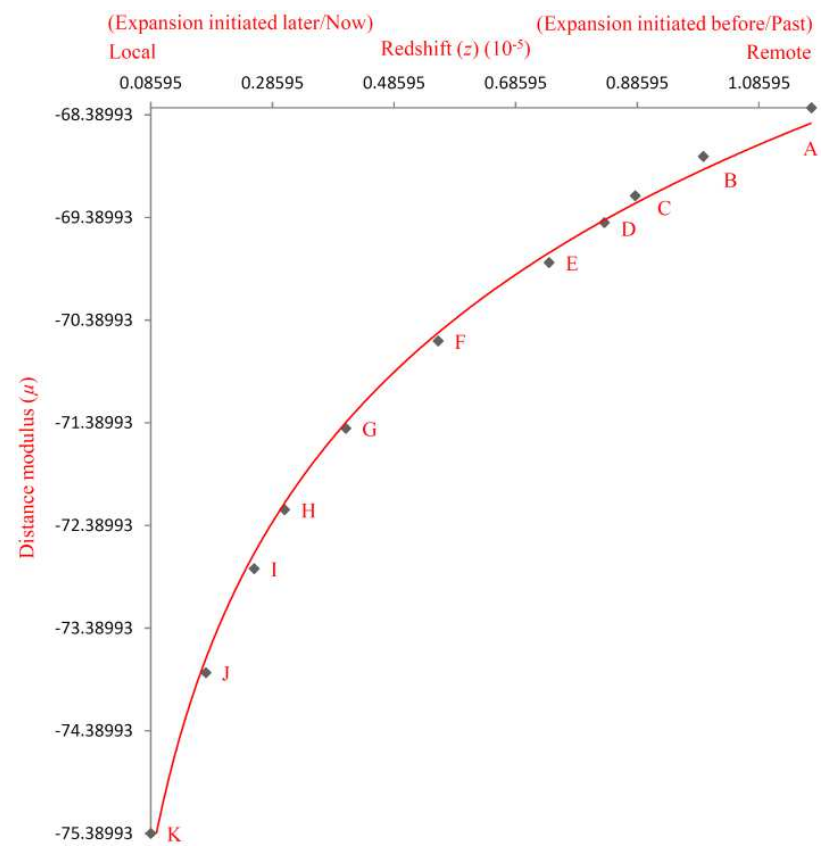

Figure 21. Distance modulus versus redshift relationship for 11 test particles (local and remote particles from Figure 5) is consistent with an accelerating Universe (in agreement with Figure 20) when remote particles with high redshifts began expanding before the expansion got initiated for local particles with low redshifts.

\subsection{Replication of results and its importance in science}

Replication of results plays an important role in scientific analysis. If same results are obtained after conducting an analysis on the original research, then the analysis is most likely to be correct.

Having considered 11 test particles to prove the credibility of the study conducted in this paper that happens to be most consistent with consecutive expansion epochs of the Universe that preceded the current expansion epoch, it would now be imperative to bring in another set of test particles to further confirm upon this undiscovered aspect that mimics cosmic acceleration so perfectly well.

We will consider another set of 113 test particles that expand consecutively - one particle after another - in agreement with consecutive expansion. All test particles have been assigned random recession velocities that decrease from remote particles to local particles (similar to what was incorporated in Section 6.1 for 11 test particles). Consecutive expansion of these 113 test particles is observed for 2 seconds with every test particle expanding consecutively after an interval of 0.01 second.

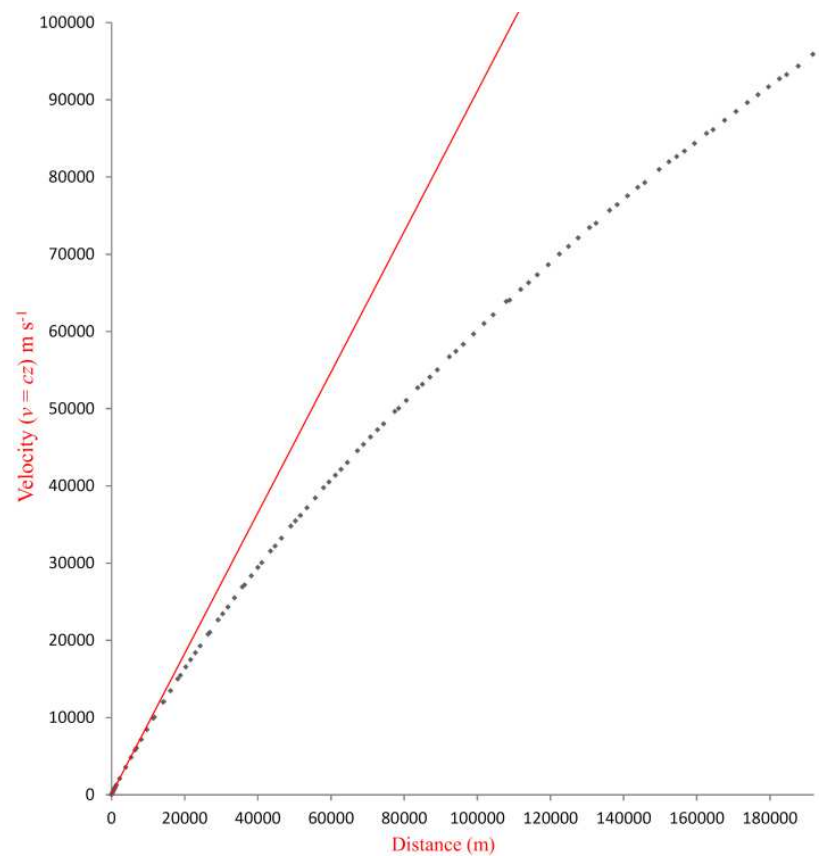

Figure 22. Velocity-distance relationship for 113 test particles (local and remote particles) expanding consecutively (one particle after another). Distances to remote particles are larger than expected with respect to local particles without acceleration. In other words, expansion initiated for remote particles before it did for local particles. 
Figure 22 depicts the velocity-distance relationship for 113 test particles undergoing consecutive expansion. Highrecession-velocity test particles that began expanding before as a consequence of consecutive expansion are further away than expected - these are the remote particles. The maximum recession velocity of one such remote particle (P1) is 95874.25 $\mathrm{m} \mathrm{s}^{-1}$ - this is the most distant particle in Figure 22.

Out of 113 test particles, last 13 test particles with low recession velocities (ranging between $199.478 \mathrm{~m} \mathrm{~s}^{-1}$ to $1 \mathrm{~m} \mathrm{~s}^{-1}$ ) were the last particles to expand, they expanded exactly at the same time and were observed only for 1 second - these are the local particles and they exhibit a linear velocity-distance relationship as they expanded exactly at the same time - the value of slope and thus the expansion rate for these last 13 test particles is the same (P101 to P113).

As shown in Figure 22, the velocity-distance relationship for 113 test particles is sufficiently self-explanatory - a plot obtained only through consecutive expansion of particles, rather than acceleration or deceleration. Remote particles that began expanding before are not only further away than expected, but they are also yielding a slower rate of expansion (deceleration) even with high recession velocities as compared to local particles that are yielding a faster rate of expansion (acceleration) even with low recession velocities. For instance, the most distant remote particle (P1) in Figure 22 with a whopping recession velocity of $95874.25 \mathrm{~m} \mathrm{~s}^{-1}$ yields a slower expansion rate of just $0.5 \mathrm{~m} \mathrm{~s}^{-1} \mathrm{~m}^{-1}$, whereas a local particle (P113) in Figure 22 with minuscule recession velocity of just $1 \mathrm{~m} \mathrm{~s}^{-1}$ ends up yielding a faster expansion rate of $1 \mathrm{~m} \mathrm{~s}^{-1} \mathrm{~m}^{-1}$.

How can it scientifically be justified that high recession velocity of $95874.25 \mathrm{~m} \mathrm{~s}^{-1}$ implies deceleration or a slower rate of expansion, whereas low recession velocity of $1 \mathrm{~m} \mathrm{~s}^{-1}$ implies a faster rate of expansion or acceleration? This paradoxical attribute is not something that can so easily be neglected and swept under the rug. With scientific responsibility one should be equipped with an explanation to such a paradoxical trend.

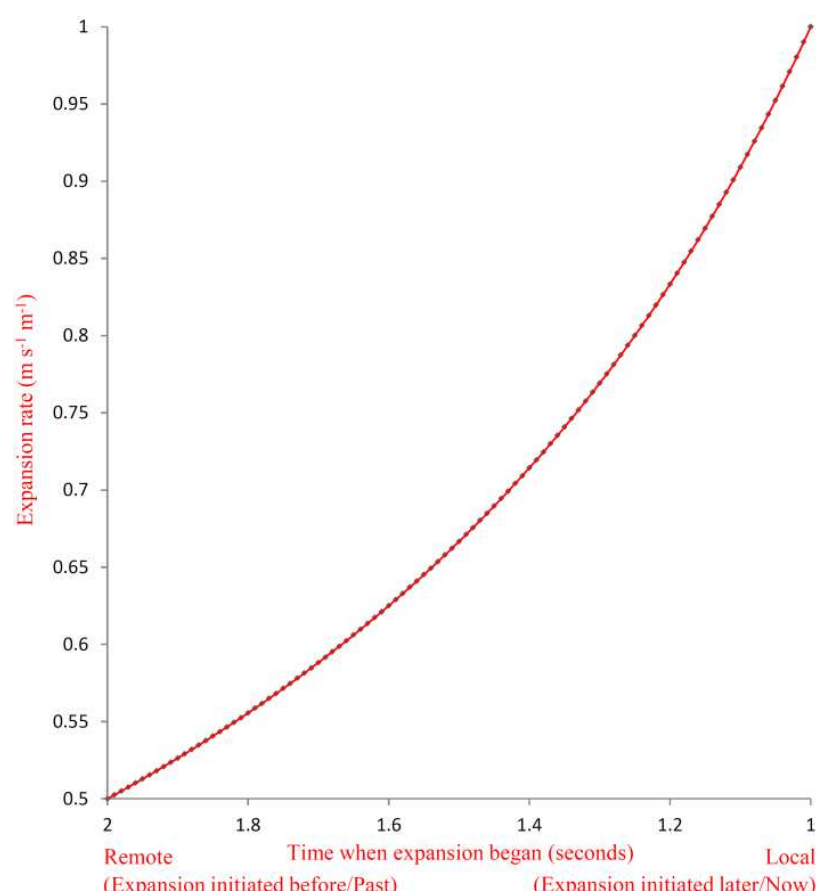

Figure 23. Plot of expansion rate versus time when expansion began (measured from past to present) for 113 test particles (remote and local particles from Figure 22) mimics an accelerating expansion (expansion rate appears to be increasing with time) when remote particles with high recession velocities began expanding before the expansion got initiated for local particles with low recession velocities. Expansion rate for remote particles that are further away than expected (see Figure 22) is lower even with high recession velocities as compared to the expansion rate for nearby local particles even with low recession velocities.

Plot of expansion rate versus time for 113 test particles as shown above in Figure 23 gives an overall and much clearer picture how the expansion rate appears to have increased over time from past to present (remote to local) without having subjected any of those test particles to deceleration or acceleration. The plot is similar to what we have already witnessed for 11 test particles in Figure 7 and for 5 type Ia supernovae in Figure 6.

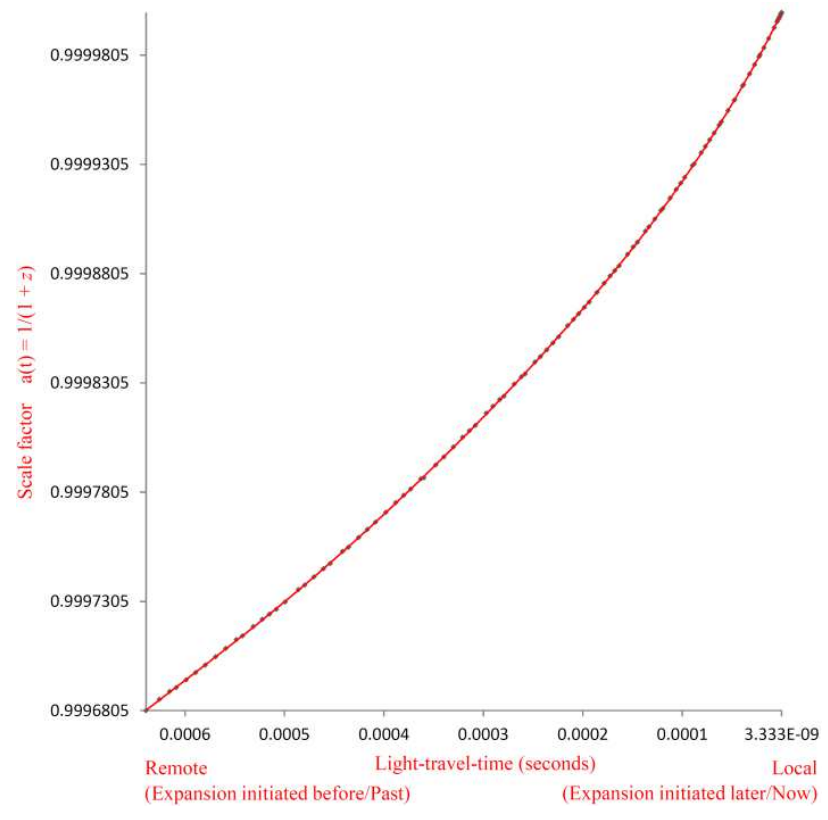

Figure 24. Plot of scale factor versus light-travel-time (measured from past to present) for 113 test particles (remote and local particles from Figure 22) mimics an expansion that accelerates (scale factor increasing with time) when remote particles with high redshifts began expanding before the expansion got initiated for local particles with low redshifts. Figure is consistent with Universe that is accelerating as scale factor is increasing with time.

Scale factor evolution for 113 test particles shown above in Figure 24 is consistent with an expansion that is accelerating over time from past to present (remote to local). Surprisingly, not even a single test particle was subjected to deceleration or acceleration.

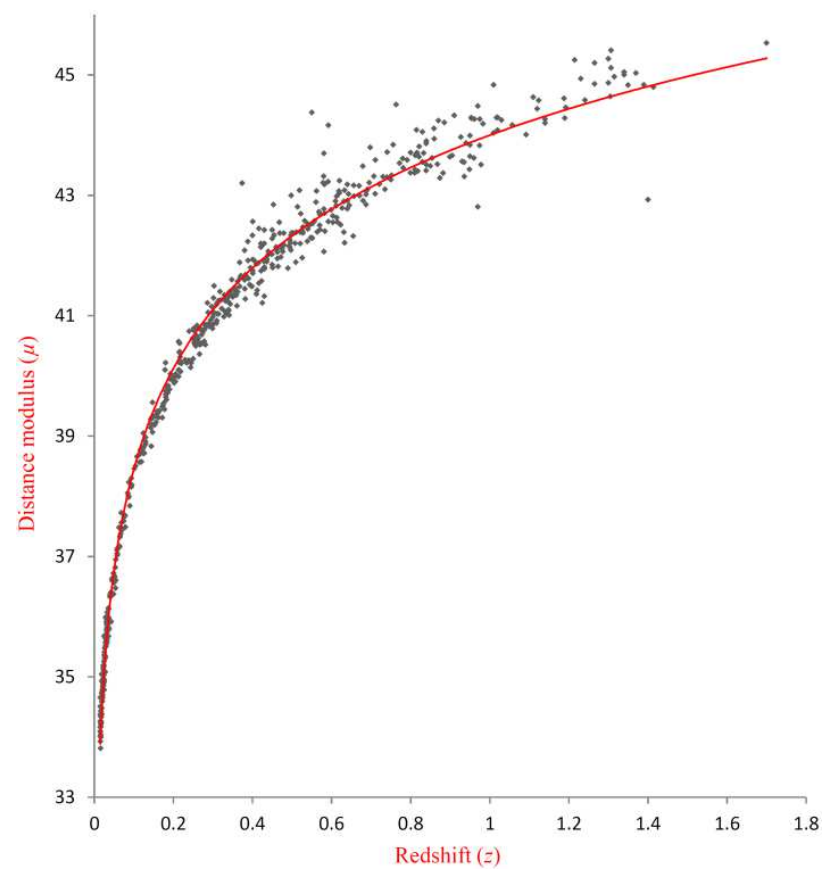

Figure 25. Distance modulus versus redshift relationship for 588 type Ia supernovae (local and remote supernovae from Figure 1). The deviation from linearity at high redshifts suggests that type Ia supernovae are further away than expected, this confirms the presence of an unknown energy component responsible for accelerating the Universe's expansion, thereby placing remote supernovae further away than expected.

As shown above in Figure 25, distance modulus versus redshift relationship has become the new Hubble diagram in cosmic literature. The plot is linear at low redshifts before curving logarithmically upwards. The deviation from linearity at high redshifts clearly suggests that remote type Ia supernovae are further away than expected due to cosmic acceleration.

In Figure 26, distance modulus versus redshift relationship has been plotted for 113 test particles undergoing consecutive expansion, the plot is similar to what has been plotted for 588 type Ia supernovae here in Figure 25 - the plot is linear at low redshifts before curving logarithmically upwards. The deviation from linearity at high redshifts clearly suggests that remote 
particles that began expanding before as per consecutive expansion are further away than expected; not even a single test particle was subjected to acceleration or deceleration.

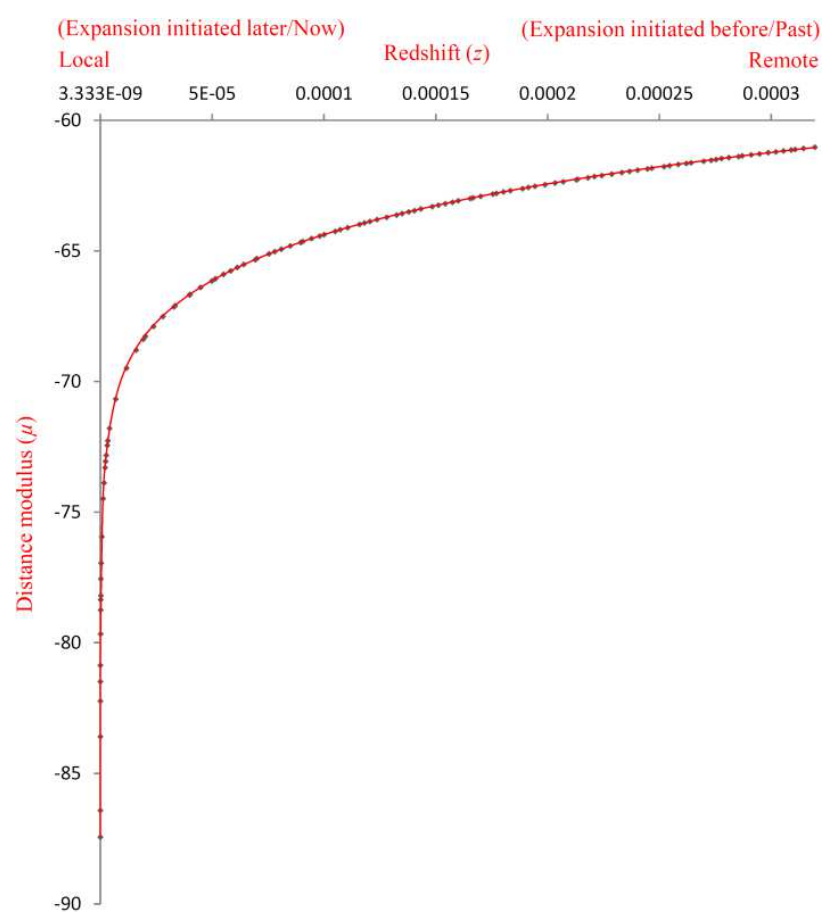

Figure 26. Distance modulus versus redshift relationship for 113 test particles (local and remote particles from Figure 22) is consistent with an accelerating Universe (in agreement with Figure 25) when remote particles with high redshifts began expanding before the expansion got initiated for local particles with low redshifts.

Perhaps we should introduce more random recession velocities for a few random remote particles. The resultant velocity-distance relationship for 113 test particles in such scenario turns out to be as shown below in Figure 27.

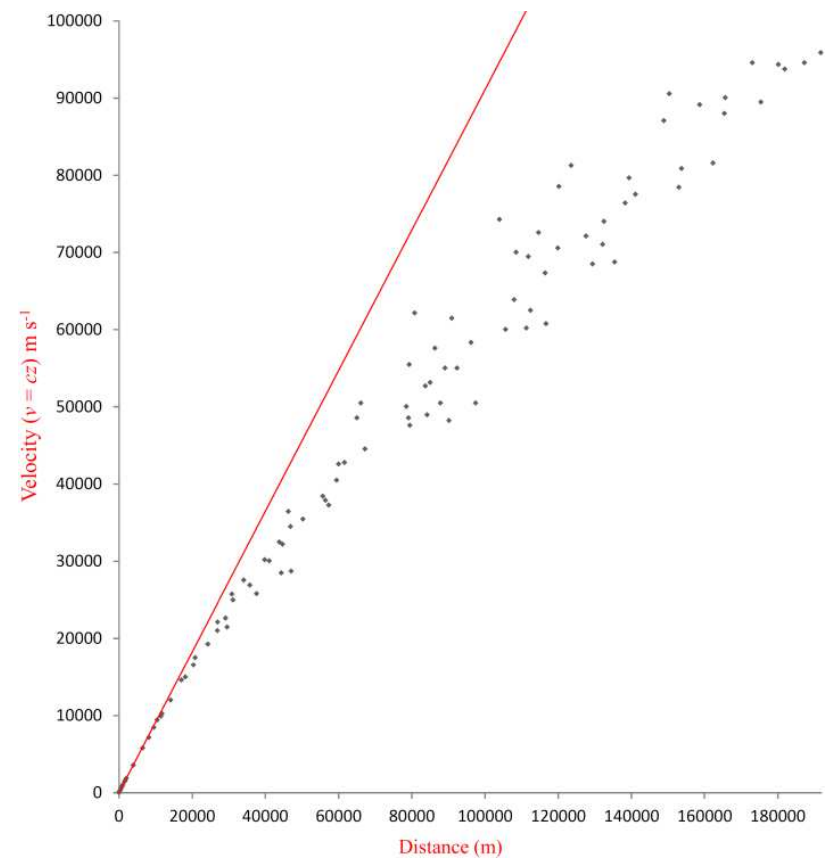

Figure 27. Velocity-distance relationship for 113 test particles (local and remote particles) expanding consecutively (one particle after another). Recession velocities have been made more random for a few randomly-targeted remote particles. Distances to remote particles are still larger than expected with respect to local particles without acceleration. In other words, expansion initiated for remote particles before it did for local particles.

Figure 27 is still consistent with consecutive expansion of particles - high-recession-velocity remote particles began expanding before the expansion got initiated for low-recessionvelocity local particles. Overall recession velocities are still increasing with distances, however, there is more randomness now in recession velocities and hence the distances to a few random remote particles. Such randomness has made the plot much similar to the redshift-distance relationship for 588 type Ia supernovae plotted in Figure 1. Local test particles that expanded exactly at the same time and were observed only for 1 second still continue exhibiting a linear velocity-distance relationship consistent with nearby local supernovae.

Although random recession velocities were introduced for a few randomly-targeted remote particles, the plot of expansion rate versus time relationship for these 113 test particles still remains conserved as shown below in Figure 28.

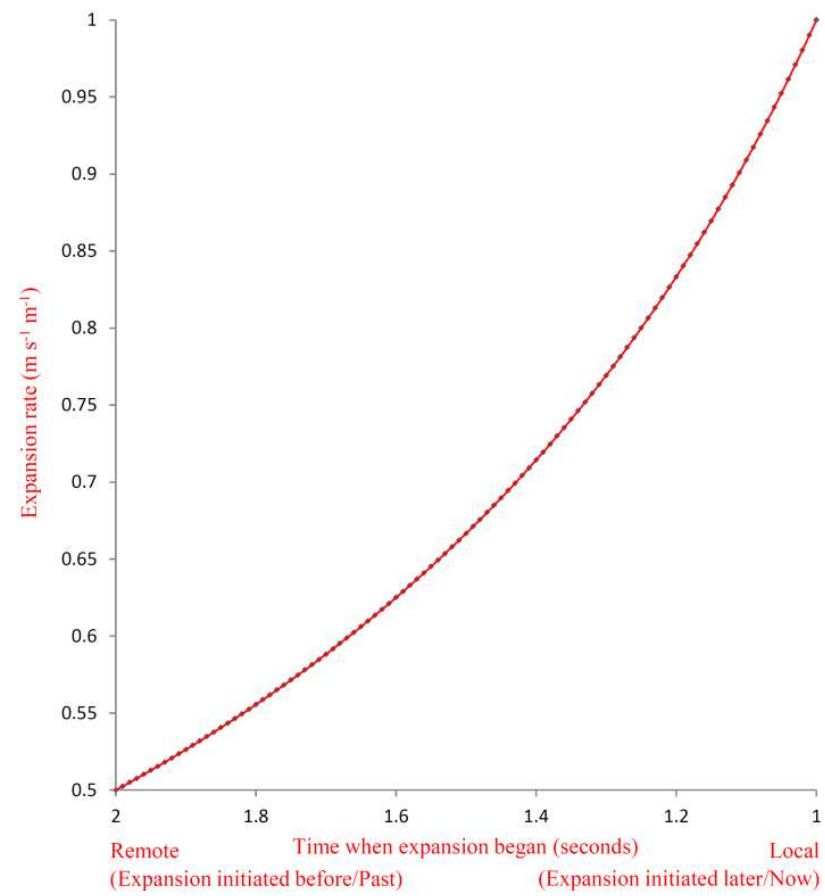

Figure 28. Plot of expansion rate versus time when expansion began (measured from past to present) for 113 test particles (remote and local particles from Figure 27) still mimics an accelerating expansion (expansion rate appears to be increasing with time) when remote particles with high recession velocities began expanding before the expansion got initiated for local particles with low recession velocities. Expansion rate for remote particles that are further away than expected (see Figure 27) is lower even with high recession velocities as compared to the expansion rate for nearby local particles even with low recession velocities.

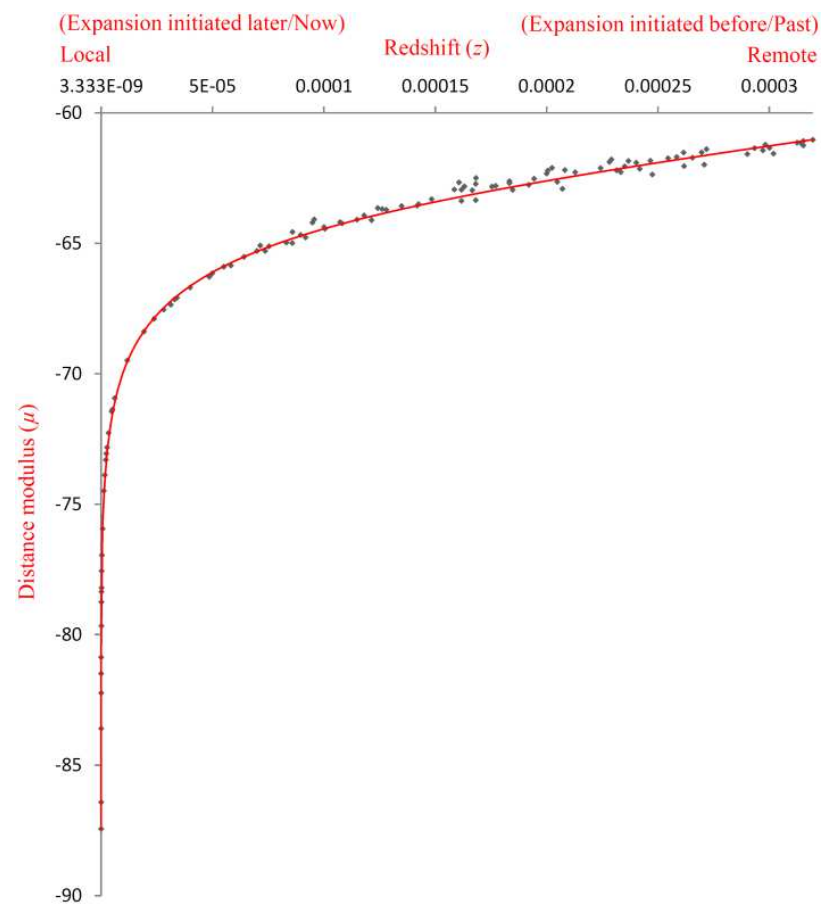

Figure 29. Distance modulus versus redshift relationship for 113 test particles (local and remote particles from Figure 27) is still consistent with an accelerating Universe (in agreement with Figure 25) when remote particles with high redshifts began expanding before the expansion got initiated for local particles with low redshifts.

After introducing more random recession velocities for a few randomly-targeted remote particles, distance modulus versus redshift relationship for 113 test particles as shown above in Figure 29 has also become much similar to distance modulus versus redshift relationship for 588 type Ia supernovae plotted in Figure 25. Figure 29 is still consistent with consecutive 
expansion of particles, rather than acceleration or deceleration of those test particles - the plot is consistently linear at low redshifts before curving logarithmically upwards.

Having already plotted scale factor versus time relationship for 5 type Ia supernovae (Figure 12), 11 test particles (Figure 13 and Figure 14), and 113 test particles (Figure 24), we shall now consider plotting scale factor versus distance relationship for 588 type Ia supernovae from Figure 1 and then the same relationship for 113 test particles from Figure 27. This will help us confirm the results based on another aspect altogether.

Since distances provide an estimate of the past and the present, therefore, the scale factor evolution with distance should also help us taking a look into how the scale factor has evolved from past to present (remote to local).

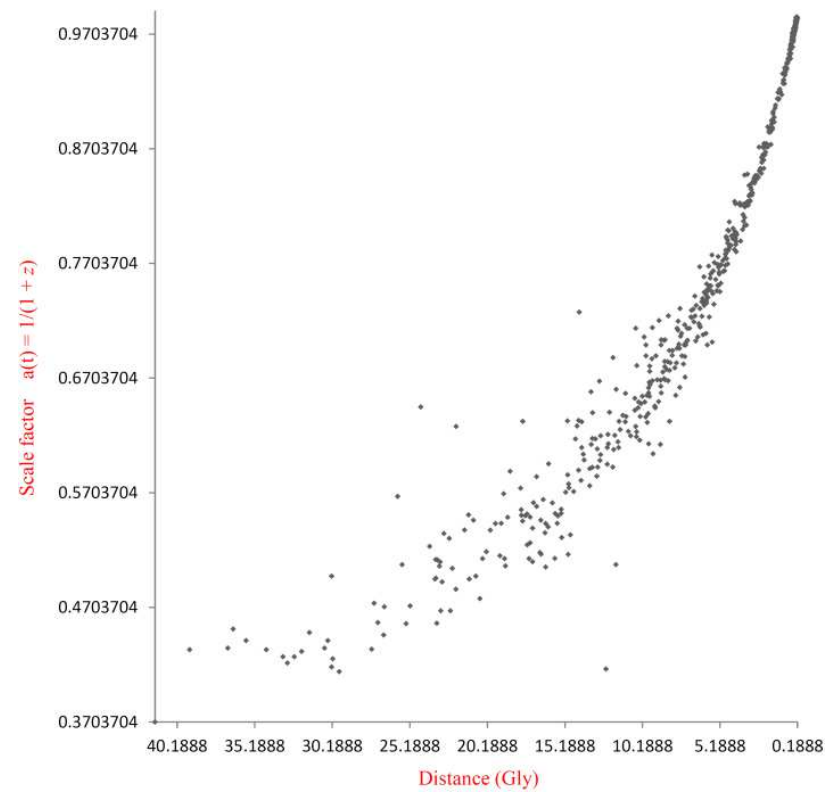

Figure 30. Plot of scale factor versus distance (distance giving an estimate of scale factor evolution from past to present - remote to local) for 588 type Ia supernovae (remote and local supernovae from Figure 1) shows an accelerating expansion (scale factor is increasing over time).

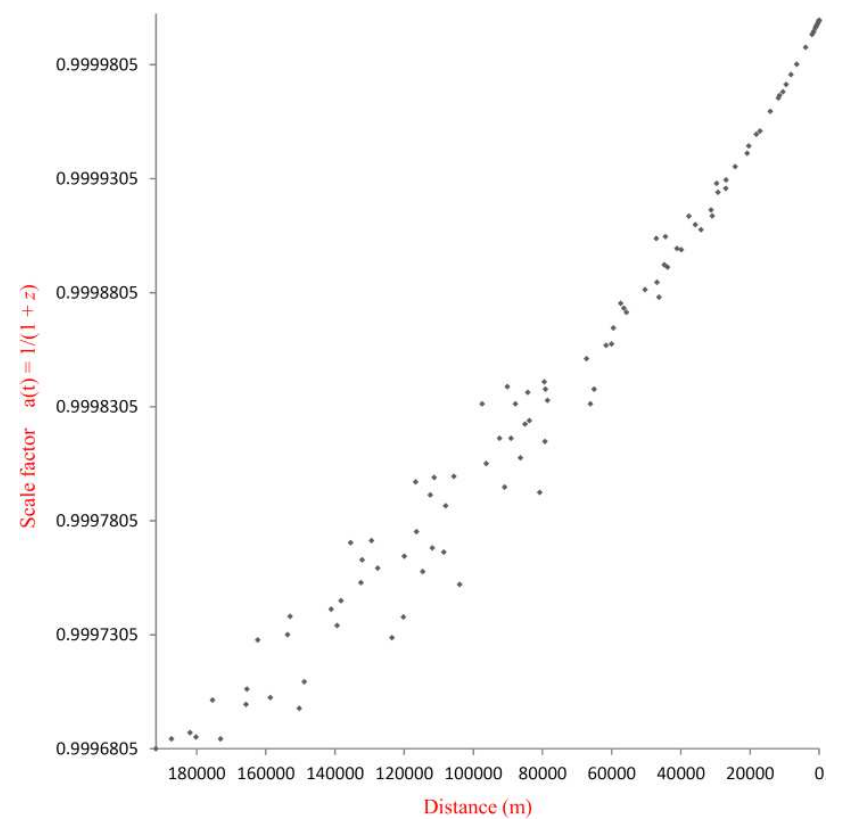

Figure 31. Plot of scale factor versus distance (distance giving an estimate of scale factor evolution from past to present - remote to local) for 113 test particles (remote and local particles from Figure 27) also shows an accelerating expansion (scale factor is increasing over time) when remote particles with high redshifts began expanding before the expansion got initiated for local particles with low redshifts.

As depicted above in Figure 30 and Figure 31, the scale factor evolution from past to present shows a prominent increase in scale factor in the recent epoch. For 588 type Ia supernovae this suggests a recent accelerated expansion. A similar relationship obtained for 113 test particles should also suggest the same - a recent accelerated expansion. However, the scale factor versus distance relationship for 113 test particles (Figure 31) has been obtained through consecutive expansion of those test particles, and not through deceleration or acceleration. This should again make it clear enough that such relationship can also be obtained through consecutive expansion epochs of the Universe that preceded the current expansion epoch, rather than cosmic deceleration that preceded the current epoch of cosmic acceleration.

\subsection{Deceleration parameter}

Measuring the deceleration parameter $\left(q_{0}\right)$ helps probing the expansion history of the Universe. We can obtain the deceleration parameter by using the following approximation,

$$
q_{0} \approx-\frac{2 D H-2 c z-c z^{2}}{c z^{2}}
$$

where $H$ is the expansion rate $\left(\mathrm{m} \mathrm{s}^{-1} \mathrm{~m}^{-1}\right), D$ is the distance $(\mathrm{m})$, $c$ is the velocity of light $\left(\mathrm{m} \mathrm{s}^{-1}\right)$, and $z$ is the redshift. The above equation for deceleration parameter $\left(q_{0}\right)$ is valid for $z<0.5$ and has been obtained using the following approximation from Schmidt (2012).

$$
D_{L} \approx \frac{c}{H_{0}}\left[z+z^{2} \frac{\left(1-q_{0}\right)}{2}\right]
$$

The surprising transition of Universe's expansion from deceleration to acceleration has already shown that the deceleration parameter, as per the "current acceleration of the expansion", is negative, that is, $q_{0}<0$ (Riess et al. 1998).

We have already obtained the expansion rate versus time relationship for 5 type Ia supernovae in Section 6.2 (Figure 6) we get an overall picture how the expansion rate has increased over time (transition from deceleration to acceleration - remote to local - past to present).

In this section we will see how the deceleration parameter values vary for the remote type Ia supernova (SN 1995K) while using evolving values of the expansion rate $(H)$ obtained from 4 type Ia supernovae (remote to local - past to present), we will then see if the most distant remote test particle from Figure 22 (particle P1) also exhibits a similar attribute for the deceleration parameter, that is, $q_{0}<0$ while using more recent or evolving values of the expansion rate $(H)$.

\section{Table 1}

Deceleration parameter $\left(q_{0}\right)$ values for the remote supernova (SN $1995 \mathrm{~K}$ ) obtained by using evolving values of expansion rate $(H)$ (past to present - remote to local).

\begin{tabular}{lcr}
\hline \hline SN & $H\left(\mathrm{~km} \mathrm{~s}^{-1} \mathrm{Mpc}^{-1}\right)$ & $q_{0}$ values for SN 1995K \\
\hline $1995 \mathrm{~K}$ & 48.1919 & +1.000003756 \\
1999DU & 53.0730 & +0.577103695 \\
1992BS & 59.9955 & -0.022663912 \\
1994S & 69.0543 & -0.807521234 \\
\hline
\end{tabular}

Table 1 represents the expansion rate for 4 type Ia supernovae (remote to local) and the deceleration parameter values for the remote type Ia supernova SN $1995 \mathrm{~K}(z=0.479, D=9.7211$ Gly). The deceleration parameter is getting "more negative" while using evolving values of $H$ obtained from 4 type Ia supernovae (evolving values of $H$ signify how the expansion rate has increased over time from remote to local - past to present, while the departure of deceleration parameter from positive $\left(q_{0}>0\right)$ to increasingly negative values $\left(q_{0}<0\right)$ suggests transition of Universe's expansion from deceleration to acceleration).

In a Universe that is dominated by matter, one would rightfully expect the expansion rate to slow down with time due to gravitational attraction of matter, in such case, the deceleration parameter would simply be given as,

$$
q_{0}=\frac{\Omega_{M}}{2}
$$

where $\Omega_{M}$ denotes the matter content/matter density. However, the increasingly negative values for deceleration parameter obtained for the remote supernova (SN 1995K) while using more recent or evolving values of the expansion rate $(H)$ - from past to present (remote to local) is disturbing as it necessitates the introduction of "negative mass" exhibiting anti-gravitational effect that acts against the gravitational attraction of matter and accelerates the expansion of the Universe. According to Riess (2012), "The only way to match the change in the expansion rate was to allow the Universe to have a "negative" mass". 


\section{Karan R.Takkhi}

Now, since negative mass is practically impossible, therefore, the only solution to quench this impossible paradigm of negative mass was to reintroduce the abandoned cosmological constant " $\Lambda$ ". "From our working definition of $q_{0}$, negative values for the current deceleration (i.e., accelerations) are generated only by a positive cosmological constant and not from unphysical, negative mass density" (Riess et al. 1998). Consequently, the significance of the cosmological constant was calculated, "99.7\% to $99.8 \%$ confidence no matter what the mass density" (Riess 2012). Therefore, Equation (14) now gets written as,

$$
q_{0}=\frac{\Omega_{M}}{2}-\Omega_{\Lambda}
$$

where $\Omega_{\Lambda}$ denotes the energy content/energy density - the history of this takes us back into the past. In 1917, Einstein had introduced the cosmological constant into his gravitational field equations to represent the energy associated with empty space. The term was necessary to account for a "static Universe" a Universe that neither contracts nor expands; the distance between objects remains the same in such static Universe. Cosmological constant was introduced to overcome the gravitational attraction of matter that would otherwise cause the Universe to contract and collapse under gravity.

However, in 1929, the revolutionizing discovery by Hubble from his observations of distant galaxies through the 100-inch Hooker telescope atop Mount Wilson in California proved that the Universe was expanding; it was not at all "static" as was previously being considered. This observation of expanding Universe had led to the abandoning of the cosmological constant.

The cosmological constant denotes the energy density of empty space or vacuum (vacuum energy density). However, when the value of the cosmological constant is obtained according to the quantum field theory, a huge discrepancy is introduced - a discrepancy involving a mismatch by 120 orders of magnitude $\left(10^{120}\right)$. Such discrepant value of the cosmological constant would lead to a vacuum catastrophe.

Having obtained the increasingly negative values for the deceleration parameter for the remote supernova (SN 1995K) by using evolving values of expansion rate $(H)$ obtained from 4 type Ia supernovae (remote to local - past to present), we will now bring the test particles into picture and study how the deceleration parameter $\left(q_{0}\right)$ values vary for the remote particle (P1) while using evolving values of expansion rate $(H)$ obtained from 4 test particles (remote to local - past to present) when test particles expanded consecutively - one particle after another as per consecutive expansion.

We have already obtained the expansion rate versus time relationship for 11 test particles in Section 6.2 (Figure 7) and then for 113 test particles in Section 6.9 (Figure 23) - we get an overall picture how the expansion rate appears to have increased over time (transition from deceleration to acceleration - remote to local - past to present) without having subjected any of those test particles to deceleration or acceleration.

Since we have not subjected any test particle to deceleration or acceleration, therefore, it would be very interesting to see if such similar feat would also be traced out by the most distant remote test particle (P1) (Figure 22), that is, $q_{0}<0$ while using more recent or evolving values of the expansion rate $(H)$. A similar behaviour would help us confirm the study conducted in this paper to an unprecedented level that supports, not acceleration, but consecutive expansion epochs of the Universe that preceded the current expansion epoch.

Table 2

Deceleration parameter $\left(q_{0}\right)$ values for the remote particle (P1) obtained by using evolving values of expansion rate $(H)$ (past to present - remote to local).

\begin{tabular}{lcc}
\hline \hline Particle & $H\left(\mathrm{~m} \mathrm{~s}^{-1} \mathrm{~m}^{-1}\right)$ & $q_{0}$ values for P1 \\
\hline P1 & 0.5 & +1.000000000 \\
P41 & 0.625 & -1563.549397 \\
P76 & 0.8 & -3753.918553 \\
P113 & 1.0 & -6257.197589 \\
\hline
\end{tabular}

Table 2 represents the expansion rate for 4 test particles (remote to local) and the deceleration parameter values for the remote particle $\mathrm{P} 1\left(z=3.195808333 \times 10^{-4}, D=191748.5 \mathrm{~m}\right)$. It must again be noted that remote particle $\mathrm{P} 1$ is the first particle that expanded, whereas local particle P113 is the last or more recent particle that underwent expansion as per consecutive expansion.

It is very clear from Table 2 that the deceleration parameter is getting "more negative" while using evolving values of $H$ obtained from 4 test particles (evolving values of $H$ signify how the expansion rate has increased over time from remote to local - past to present, while the departure of deceleration parameter from positive $\left(q_{0}>0\right)$ to increasingly negative values $\left(q_{0}<0\right)$ suggests transition of particles' expansion from deceleration to acceleration). This is exactly what we have obtained from 4 type Ia supernovae in Table 1 .

The increasingly negative values for deceleration parameter $\left(q_{0}<0\right)$ obtained for the remote test particle $(\mathrm{P} 1)$ while using more recent or evolving values of the expansion rate $(H)-$ from past to present (remote to local) also necessitates the introduction of negative mass responsible for accelerating the expansion of test particles over time. Now, since there is no such thing as negative mass as already discussed, therefore, the only way to explain those increasingly negative values of deceleration parameter would be to introduce an energy component responsible for accelerating the expansion of those particles with time from past to present (remote to local). However, as we are well aware that not even a single test particle was subjected to deceleration or acceleration, therefore, the presence of an energy component responsible for the increasingly negative values of deceleration parameter, thereby indicating current acceleration of the expansion is again completely out of question. It is only because of consecutive expansion of particles wherein expansion of one particle is followed by the next in a consecutive manner that we are getting increasingly negative values for deceleration parameter for the remote test particle (P1) while using more recent or evolving values of the expansion rate $(H)$ - from past to present (remote to local).

\subsection{Reproducibility of results and its importance in science}

Not just replication, but reproducibility of results also plays an important role in scientific analysis. If "same results are obtained again" after conducting an analysis on the original research, then reproducibility along with replication of those results adds further credence to the analysis and helps warrant its validity.

Having considered 11 test particles and then 113 test particles to prove the validity of the study conducted in this paper that happens to be most consistent with "consecutive expansion epochs of the Universe that preceded the current expansion epoch", rather than "cosmic deceleration that preceded the current epoch of cosmic acceleration", we will now bring in another set of test particles to further confirm upon this undiscovered aspect that mimics cosmic acceleration so perfectly well.

We will consider a set of 400 test particles that expand consecutively - one particle after another - in agreement with consecutive expansion. This time we will assign the redshifts of 400 type Ia supernovae from Figure 1 to these 400 test particles (redshifts ranging from 0.015 to 0.498 will help keep Equation (12) and Equation (13) valid, this will also make the analysis more relatable in terms of actual redshifts observed). As observed for type Ia supernovae, the redshifts decrease from remote particles to local particles (similar to what has been incorporated so far). Consecutive expansion of these 400 test particles is observed for 4.99 seconds with every test particle expanding consecutively after an interval of 0.01 second.

Initially, particle $\mathrm{SN} 1(z=0.498)$ begins expanding, 0.01 second later, particle SN2 $(z=0.497)$ begins expanding, the expansion of particle SN2 is followed by the expansion of particle SN3 $(z=0.497)$ after another 0.01 second. Consecutive expansion of particles continues in the same way for remaining particles - SN4 $(z=0.496)$, SN5 $(z=0.495)$, SN6 $(z=0.495)$, SN7 $(z=0.493)$, and so on. Particle SN400 $(z=0.015)$ is the last particle to expand, and it is observed only for 1 second. By the time this last particle (SN400) expands and is observed for 1 second, particle SN1 has already been expanding for 4.99 seconds, particle SN2 for 4.98 seconds, particle SN3 for 4.97 seconds, and so on, this becomes their respective observation time or the expansion time. 
Figure 32 depicts the velocity-distance relationship for 400 test particles undergoing consecutive expansion. High-redshift test particles that began expanding before as a consequence of consecutive expansion are further away than expected - these are the remote particles. The maximum redshift of one such remote particle (SN1) is 0.498 - this is the most distant particle in Figure 32. Out of 400 test particles, test particle SN400 with low redshift $(z=0.015)$ was the last particle to expand and was observed only for 1 second - this is the local particle.

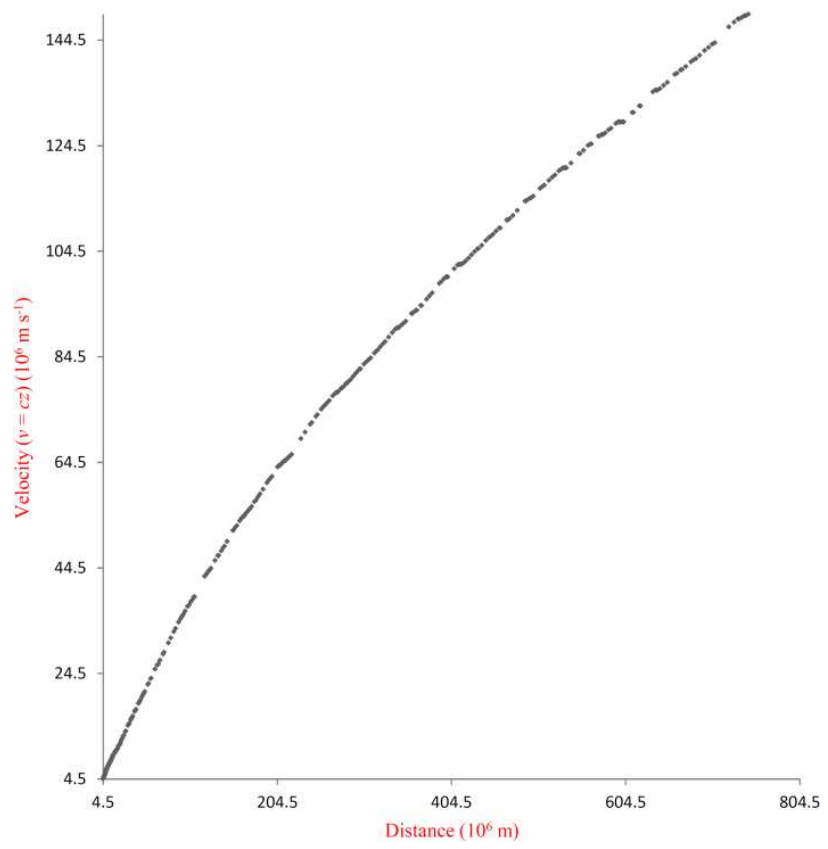

Figure 32. Velocity-distance relationship for 400 test particles (local and remote particles) expanding consecutively (one particle after another). Distances to remote particles are larger than expected with respect to local particles without acceleration. In other words, expansion initiated for remote particles before it did for local particles.

Velocity-distance relationship for 400 test particles is again sufficiently self-explanatory - a plot obtained only through consecutive expansion of particles, rather than acceleration or deceleration. Remote particles that began expanding before are not only further away than expected, but they are also yielding a slower rate of expansion (deceleration) even with high recession velocities as compared to local particles that are yielding a faster rate of expansion (acceleration) even with low recession velocities as shown below.

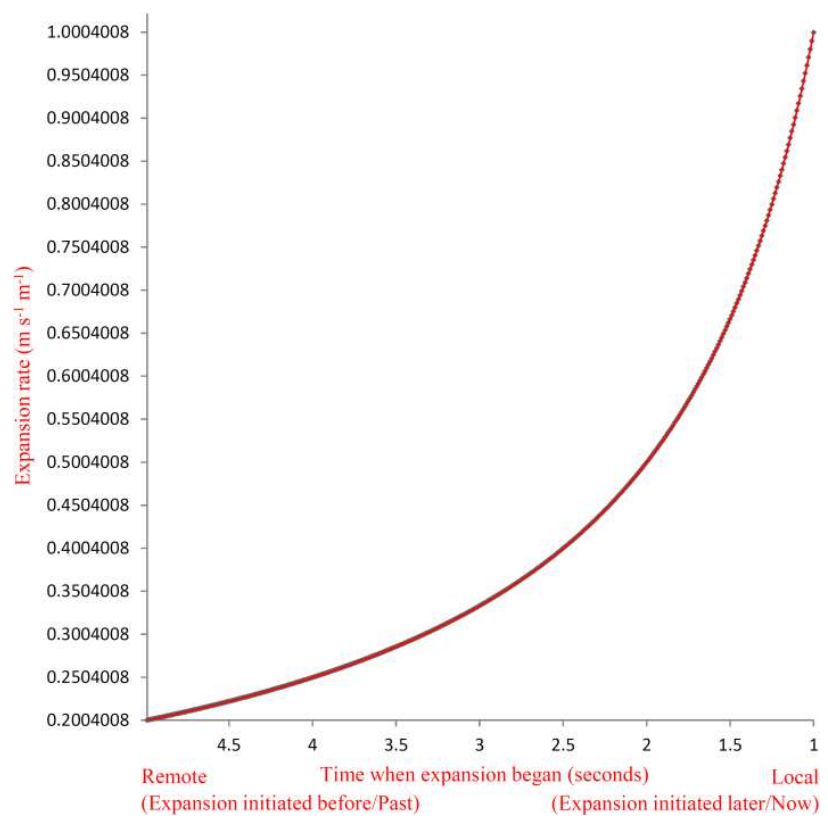

Figure 33. Plot of expansion rate versus time when expansion began (measured from past to present) for 400 test particles (remote and local particles from Figure 32) mimics an accelerating expansion (expansion rate appears to be increasing with time) when remote particles with high recession velocities began expanding before the expansion got initiated for local particles with low recession velocities. Expansion rate for remote particles that are further away than expected (see Figure 32) is lower even with high recession velocities as compared to the expansion rate for nearby local particles even with low recession velocities.
Plot of expansion rate versus time for 400 test particles as shown in Figure 33 gives an overall and much clearer picture how the expansion rate appears to have increased over time from past to present (remote to local) without having subjected any of those test particles to deceleration or acceleration. The plot is similar to what we have already witnessed for 11 test particles in Figure 7, for 5 type Ia supernovae in Figure 6, and for 113 test particles in Figure 23, and Figure 28.

We need to ask ourselves an important question here to which we already know the answer - has the expansion rate really increased for test particles over time from past to present (remote to local) as a consequence of accelerated expansion, or is it that the expansion rate "appears" to have increased for test particles over time as a consequence of consecutive expansion?

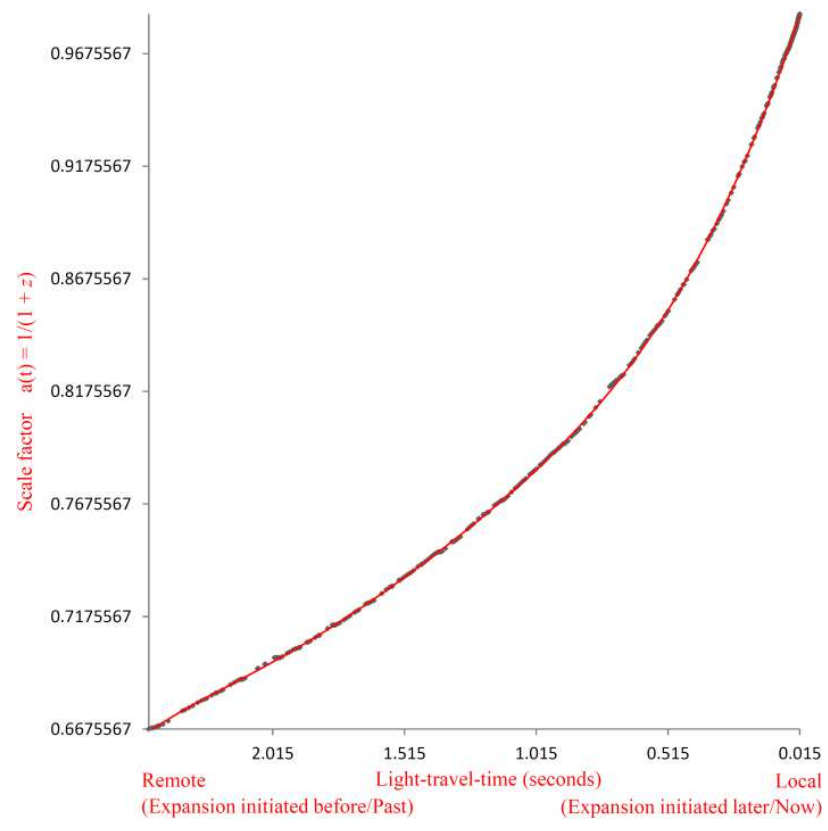

Figure 34. Plot of scale factor versus light-travel-time (measured from past to present) for 400 test particles (remote and local particles from Figure 32) mimics an expansion that accelerates (scale factor increasing with time) when remote particles with high redshifts began expanding before the expansion got initiated for local particles with low redshifts. Figure is consistent with Universe that is accelerating as scale factor is increasing with time.

Scale factor evolution for 400 test particles as shown above in Figure 34 is again consistent with an expansion that is accelerating over time from past to present (remote to local) (in agreement with Figure 12). Surprisingly, not even a single test particle was subjected to deceleration or acceleration.

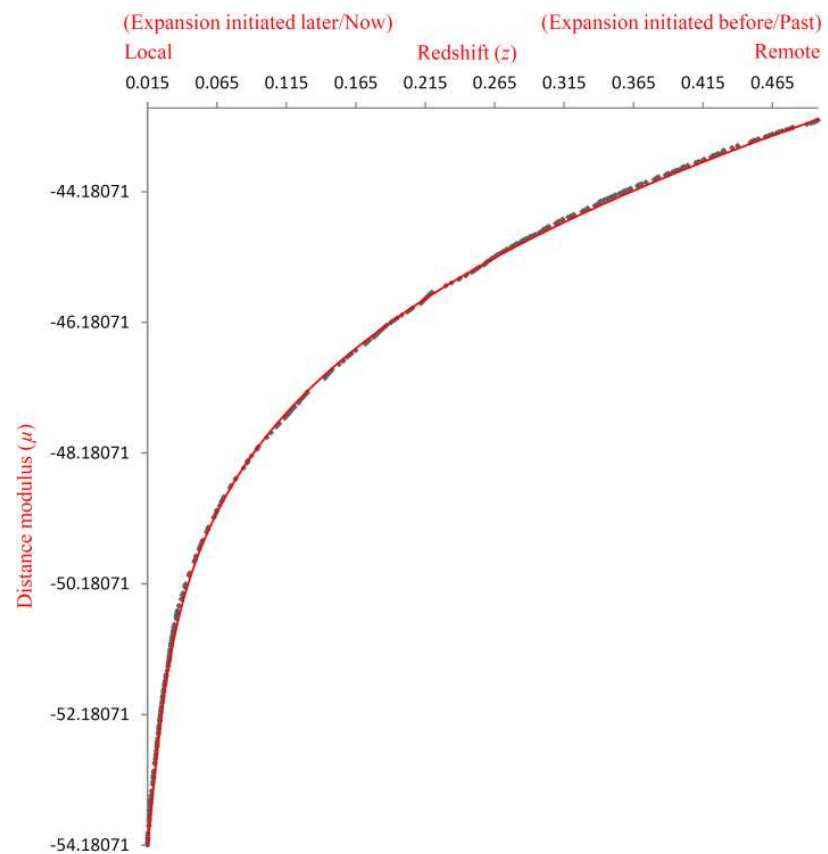

Figure 35. Distance modulus versus redshift relationship for 400 test particles (local and remote particles from Figure 32) is consistent with an accelerating Universe (again in agreement with Figure 25) when remote particles with high redshifts began expanding before the expansion got initiated for local particles with low redshifts. 


\section{Karan R.Takkhi}

As shown in Figure 35, distance modulus versus redshift relationship has been plotted for 400 test particles undergoing consecutive expansion, the plot is again similar to distance modulus versus redshift relationship for 588 type Ia supernovae plotted in Figure 25 - the plot is linear at low redshifts before curving logarithmically upwards. The deviation from linearity at high redshifts clearly suggests that remote particles that began expanding before as per consecutive expansion are further away than expected; again, not even a single test particle was subjected to acceleration or deceleration.

In Section 6.10 we witnessed the increasingly negative values for the deceleration parameter for the remote supernova (SN $1995 \mathrm{~K})$ while using evolving values of expansion rate $(H)$ obtained from 4 type Ia supernovae (remote to local - past to present), we also witnessed the increasingly negative values for the deceleration parameter for the remote test particle (P1) while using evolving values of expansion rate $(H)$ obtained from 4 test particles (remote to local - past to present).

We will now bring 400 test particles into picture and study how the deceleration parameter $\left(q_{0}\right)$ values vary for the remote particle (SN5) while using evolving values of expansion rate $(H)$ obtained from 8 test particles (remote to local - past to present) when test particles expanded consecutively - one particle after another as per consecutive expansion.

We have already obtained the expansion rate versus time relationship for 400 test particles in this section (Figure 33) we get an overall picture how the expansion rate appears to have increased over time (transition from deceleration to acceleration - remote to local - past to present) without having subjected any of those test particles to deceleration or acceleration.

Since we have not subjected any of those 400 test particles to deceleration or acceleration, therefore, it would be very interesting to see again if such similar feat would be traced out by the fifth most distant remote test particle (SN5) (Figure 32), that is, $q_{0}<0$ while using more recent or evolving values of the expansion rate $(H)$. A similar behaviour if obtained again will further help us confirm the study conducted in this paper to an unprecedented level that supports "consecutive expansion epochs of the Universe that preceded the current expansion epoch", rather than "cosmic deceleration that preceded the current epoch of cosmic acceleration".

Table 3

Deceleration parameter $\left(q_{0}\right)$ values for the remote particle (SN5) obtained by using evolving values of expansion rate $(H)$ (past to present remote to local).

\begin{tabular}{llr}
\hline \hline Particle & $H\left(\mathrm{~m} \mathrm{~s}^{-1} \mathrm{~m}^{-1}\right)$ & $q_{0}$ values for SN5 \\
\hline SN5 & 0.202020202 & +1.00000000 \\
SN100 & 0.25 & +0.04040404 \\
SN180 & 0.3125 & -1.20959596 \\
SN250 & 0.4 & -2.95959596 \\
SN300 & 0.5 & -4.95959596 \\
SN340 & 0.625 & -7.45959596 \\
SN375 & 0.8 & -10.95959596 \\
SN400 & 1.0 & -14.95959596 \\
\hline
\end{tabular}

Table 3 represents the expansion rate for 8 test particles (remote to local) and the deceleration parameter values for the remote particle SN5 $(z=0.495, D=735075000 \mathrm{~m})$. It must again be noted that remote particle SN5 is the fifth particle that expanded, whereas local particle P400 is the last or more recent particle that underwent expansion as per consecutive expansion.

It is again clear from Table 3 that the deceleration parameter is getting "more negative" while using evolving values of $H$ obtained from 8 test particles (evolving values of $H$ signify how the expansion rate has increased over time from remote to local - past to present, while the departure of deceleration parameter from positive $\left(q_{0}>0\right)$ to increasingly negative values $\left(q_{0}<0\right)$ suggests transition of particles' expansion from deceleration to acceleration). This is exactly what we have obtained from 4 type Ia supernovae in Table 1 .

According to Equation (14), if $q_{0}$ is negative, then $\Omega_{M}$ also gets rendered negative while solving for it. Therefore, the increasingly negative values for deceleration parameter $\left(q_{0}<0\right)$ obtained for the remote test particle (SN5) while using more recent or evolving values of the expansion rate $(H)$ - from past to present (remote to local) again necessitates the introduction of negative mass responsible for accelerating the expansion of test particles over time. Negative mass being practically impossible, therefore, the only way to account for those increasingly negative values of the deceleration parameter $\left(q_{0}\right)$ would be to consider the presence of an overwhelming amount of cosmological constant or the energy associated with empty space (Equation (15)) that has accelerated the expansion of particles over time.

However, as we are well aware that not even a single test particle was subjected to deceleration or acceleration, therefore, the presence of an overwhelming amount of cosmological constant responsible for the increasingly negative values of deceleration parameter, thereby indicating current acceleration of the expansion of those particles is again completely out of question. It is only because of consecutive expansion of particles wherein expansion of one particle is followed by the next in a consecutive manner that we are getting increasingly negative values for deceleration parameter for the remote test particle (SN5) while using more recent or evolving values of the expansion rate $(H)$ - from past to present (remote to local).

As was already discussed in Section 4, if there can be an epoch of "cosmic deceleration that preceded the current epoch of cosmic acceleration" (Riess et al. 2004), then there can also be epochs of "consecutive expansion that preceded the current epoch of cosmic expansion", in fact, all similarities encountered so far in this paper for test particles and for type Ia supernovae (replication and reproduction of results) help confirming that remote structures began expanding before the expansion got initiated for local structures - the study conducted in this paper using test particles is therefore most consistent with consecutive expansion epochs of the Universe that preceded the current expansion epoch, rather than cosmic deceleration that preceded the current epoch of cosmic acceleration.

\section{DISCUSSION AND CONCLUSIONS}

(1) Direct and the best observational evidence for an accelerating Universe comes from the comparison of redshiftdistance relationship for high and low redshift type Ia supernovae. As compared to nearby local supernovae, remote supernovae appear $10 \%$ to $25 \%$ dimmer as they are further away than expected. There are some astrophysical effects that can imitate this direct evidence for an accelerating Universe from type Ia supernovae. For instance, a pervasive screen of extragalactic grey dust could dim the supernovae (Aguirre 1999a, 1999b), thereby tricking us into believing that remote supernovae are further away than expected. Luminosity evolution of type Ia supernovae can also impact the measurements if high-redshift type Ia supernovae are intrinsically fainter as compared to the low-redshift ones (Drell et al. 2000). However, according to Riess et al. (2004), "To date, no evidence for an astrophysical origin of the apparent faintness of SNe Ia has been found (Riess 2000; Coil et al. 2000; Leibundgut 2001; Sullivan et al. 2003)".

(2) By finding type Ia supernovae at different distances and measuring their redshifts we can plot the expansion rate of the Universe against time. The redshift of a remote supernova in Figure $1(z=1.7)$ is 112 times higher than the redshift of a local supernova $(z=0.015166)$, similarly, the redshift of the most distant supernova in Figure 2, SN 1995K $(z=0.479)$ is 14.38 times higher than the redshift of a local supernova $(z=0.0333)$. The expansion rate obtained for local supernovae is higher with low redshifts as compared to the expansion rate obtained for remote supernovae with high redshifts. Since observed redshifts provide an estimate of recession or expansion velocities in order to determine the expansion rate $\left(\mathrm{km} \mathrm{s}^{-1} \mathrm{Mpc}^{-1}\right)$ of the local and the remote Universe, therefore, it is very disturbing (a conundrum) to find that low recession velocities indicate acceleration (faster rate of expansion), whereas high recession velocities indicate deceleration (slower rate of expansion).

(3) The evidence for accelerating Universe came from measuring how the expansion rate $\left(\mathrm{km} \mathrm{s}^{-1} \mathrm{Mpc}^{-1}\right)$ has changed over time. Since expansion rate for local Universe is found to be higher than the expansion rate for remote Universe, therefore, we say that the Universe is expanding faster now and had a slower expansion in the past. This apparent transition of the Universe's expansion from deceleration to acceleration (past to 
present) is explained by invoking dark energy - a mysterious and hypothetical energy of unknown origin having no explanation in fundamental physics. As pointed out by Durrer (2011), "our single indication for the existence of dark energy comes from distance measurements and their relation to redshift. Supernovae, cosmic microwave background anisotropies and observations of baryon acoustic oscillations simply tell us that the observed distance to a given redshift is larger than the one expected from the locally measured Hubble parameter".

(4) Theoretical calculation for the value of dark energy believed to be the intrinsic energy associated with empty space or the vacuum energy according to the quantum field theory results into a huge 120 -orders-of-magnitude $\left(10^{120}\right)$ discrepancy. Such colossal value of the cosmological constant would have resulted into a vacuum catastrophe. This suggests that dark energy is only introduced to account for the apparent transition of the Universe's expansion from deceleration to acceleration.

(5) One should also consider that "expansion of gas molecules into the vacuum by the virtue of vacuum energy or dark energy" has never been heard off. In fact, it is worth noting that an experiment conducted by Sabulsky et al. (2019) by using atom interferometry to detect dark energy acting on a single atom inside an ultra-high vacuum chamber showed no trace of any mysterious energy. Dark energy believed to be stronger in highvacuum environments should have easily been detected acting on a minuscule mass - a single atom.

(6) It might also be important to discuss here the conjectures focusing on inhomogeneity and anisotropy that suggest our location in a region where expansion is faster than the remote background (Räsänen 2011 and Colin et al. 2019 respectively). However, based on the velocity versus luminosity-distance relationship for type Ia supernovae (Blanchard et al. 2003, Figure 4) as well as the plot by the High-Z Supernova Search Team (Figure 3), it is very disturbing to find that further-awaythan-expected remote supernovae with high recession velocities (30\% to $60 \%$ of speed of light) yield a slower rate of expansion (deceleration) as compared to the faster rate of expansion (acceleration) for nearby local supernovae with low recession velocities (just $1 \%$ to $10 \%$ of speed of light). Confidently enough, remote expansion at $60 \%$ of speed of light is way faster than local expansion at just $1 \%$ of speed of light. Then why such a paradoxical trend should even be observed wherein remote expansion at $60 \%$ of speed of light indicates a slower rate of expansion (deceleration), whereas local expansion at just $1 \%$ of speed of light indicates a faster rate of expansion (acceleration)? Therefore, with respect to these conjectures involving inhomogeneity and anisotropy, and also with respect to the surprising discovery of accelerating Universe (the apparent transition of Universe's expansion from deceleration to acceleration), how can local expansion occurring at just $1 \%$ of speed of light be justified as an expansion faster than the remote background expansion occurring at $60 \%$ of speed of light?

(7) According to Durrer (2011), "Dark energy is very disturbing. On the one hand, the fact that such an unexpected result has been found by observations shows that present cosmology is truly data driven and not dominated by ideas that can be made to fit sparse observations". Also, according to Durrer (2011), "a small cosmological constant is so unexpected and difficult to bring into agreement with our ideas about fundamental physics that people have started to look into other possibilities"

(8) One such idea that helps taking a look into another possibility that happens to mimic cosmic acceleration has therefore been presented here. The surprising discovery of accelerating Universe is the result of an undiscovered aspect that has been unravelled in this paper. Based on the study conducted and the results obtained in this paper this undiscovered aspect perfectly mimics cosmic acceleration. Direct and best evidence in favour of this undiscovered aspect also comes from observations of actual observables wherein superluminal remote expansion (expansion $>c$ ) indicates a slower rate of expansion (deceleration) as compared to subluminal local expansion (expansion $\ll c$ ) that indicates a faster rate of expansion (acceleration) - completely counterintuitive (a paradox).

(9) It remains undiscovered that an object that begins expanding before as a consequence of consecutive expansion will not only be further away than expected, but it will also be yielding a lower value of slope (or a slower rate of expansion) even with high recession velocity as compared to an object with low recession velocity that begins expanding comparatively later. If there can be an epoch of "cosmic deceleration that preceded the current epoch of cosmic acceleration" (Riess et al. 2004), then there can also be epochs of "consecutive expansion that preceded the current epoch of cosmic expansion".

(10) Logically, an object that begins expanding before as a consequence of consecutive expansion has an utmost probability of being further away than expected; the observational fact, that such object, which happens to be further away than expected, yields a lower value of slope (or a slower rate of expansion) even with high recession velocity as compared to an object that yields a higher value of slope (or a faster rate of expansion) even with low recession velocity is the most compelling evidence in favour of this undiscovered aspect.

(11) There is absolutely no other reason for an object with high recession velocity to yield a lower value of slope (or a slower rate of expansion, thereby suggesting deceleration) and then be further away than expected as compared to an object with low recession velocity, unless it began expanding before.

(12) Plotting together the high-recession-velocity remote structures that began expanding before, and the low-recessionvelocity local structures that began expanding comparatively later, causes the Hubble diagram to deviate from linearity.

(13) Comparing the slope and thus the expansion rate of highrecession-velocity remote structures that began expanding before, with the slope and thus the expansion rate of lowrecession-velocity local structures that began expanding comparatively later, causes the high-recession-velocity remote structures to appear as if they are receding slower than expected as compared to the low-recession-velocity local structures. For this reason, the recession velocity and hence the redshift of a remote structure appears to be lower than the recession velocity and hence the redshift predicted by the Hubble's law for a local structure, that is, Universe appears to be expanding slowly in the past (remotely) and faster now (locally).

(14) An object with high recession velocity that began expanding before will be further away than expected and will appear to be decelerating, whereas an object with low recession velocity that began expanding comparatively later will appear to be accelerating.

(15) Intuitively, objects that began expanding exactly at the same time will only yield exactly the same value of slope (will fall exactly on the same line), and will therefore have exactly the same expansion rate (see Figure 5 and Figure 7 - particle J and particle $\mathrm{K}$ are the only particles that have exactly the same value of slope (they fall exactly on the same line), and have exactly the same expansion rate as they began expanding exactly at the same time). Same applies for 13 local test particles (P101 to P113) in Figure 22 and Figure 27.

(16) Velocity-distance relationship for objects (particles and supernovae) (Figure 5, Figure 22, Figure 27, and Figure 32 for particles, Figure 2, Figure 4, and Figure 17 for supernovae) shows distances to remote objects to be larger than expected. Remote objects with high recession velocities are not only further away than expected, but they are also yielding a slower rate of expansion (deceleration) as compared to the faster rate of expansion (acceleration) for local objects even with low recession velocities (apparent transition of the expansion from deceleration to acceleration)

(17) Expansion rate versus time (time when expansion began) relationship for objects (supernovae and particles) (Figure 6 for supernovae, Figure 7, Figure 23, Figure 28, and Figure 33 for particles) shows expansion rate increasing with time (past to present).

(18) Observed trends discussed here in (16) and (17) are consistent with recession/expansion velocity interpretation and are possible only when objects with high recession/expansion velocities began expanding before the expansion got initiated for objects with low recession velocities.

(19) Expansion factor versus time (time when expansion began) relationship for objects (particles and supernovae, Figure 8 and Figure 9 respectively) shows expansion factor increasing exponentially with time (past to present).

(20) Expansion factor versus light-travel-time relationship for objects (particles and supernovae, Figure 10 and Figure 11 respectively) shows expansion factor increasing exponentially with time (past to present). 
(21) Scale factor versus light-travel-time relationship for objects (supernovae and particles) (Figure 12 for supernovae, Figure 13, Figure 24, and Figure 34 for particles) shows scale factor consistent with a Universe that accelerates as scale factor is increasing with time (past to present).

(22) Scale factor versus light-travel-time relationship for objects (particles and supernovae, Figure 14 and Figure 15 respectively) shows scale factor consistent with a Universe that first decelerates, and then accelerates (past to present).

(23) Scale factor versus distance relationship for objects (supernovae and particles, Figure 30 and Figure 31 respectively) shows scale factor consistent with a Universe that accelerates as scale factor is increasing over time from past to present.

(24) Distance-redshift relationship for objects (supernovae and particles, Figure 18 and Figure 19 respectively) makes it obvious that high-redshift objects lie above the line; the deviation from linearity makes it clear enough that the distances to remote objects are larger than expected.

(25) Distance modulus versus redshift relationship for objects (supernovae and particles) (Figure 20 and Figure 25 for supernovae, Figure 21, Figure 26, Figure 29, and Figure 35 for particles) shows linearity at low redshifts before curving logarithmically upwards.

(26) Observed trends discussed here in (19), (20), (21), (22), (23), (24), and (25) are consistent with redshift interpretation and are possible only when remote objects with high redshifts began expanding before the expansion got initiated for local objects with low redshifts.

(27) Moreover, the departure of deceleration parameter from positive $\left(q_{0}>0\right)$ to increasingly negative values $\left(q_{0}<0\right)$ for the remote type Ia supernova SN 1995K (Table 1) while using more recent or evolving values of expansion rate $(H)$ obtained from 4 type Ia supernovae (remote to local - past to present) suggests previous deceleration and recent acceleration. Exact attribute, that is, the departure of deceleration parameter from positive $\left(q_{0}>0\right)$ to increasingly negative values $\left(q_{0}<0\right)$ for the remote particles - particle P1 (Table 2) and particle SN5 (Table 3) while using more recent or evolving values of expansion rate $(H)$ obtained from 4 test particles (Table 2$)$ and 8 test particles (Table 3) (remote to local - past to present) should also suggest the same - previous deceleration and recent acceleration.

(28) It might be worth pointing out again that test particles were not at all subjected to acceleration or deceleration, however, we still obtained plots and the deceleration parameter values for test particles that are consistent with an expansion that is not at all slowing down with time, but is speeding up (apparent transition from deceleration to acceleration (past to present - remote to local)). Therefore, such multiple similarities obtained for test particles and supernovae strongly confirm the study conducted in this paper that happens to be most consistent with consecutive expansion epochs of the Universe that preceded the current expansion epoch, rather than cosmic deceleration that preceded the current epoch of cosmic acceleration. In agreement with consecutive expansion, remote structures began expanding before the expansion got initiated for local structures, for this reason, remote supernovae are further away than expected as compared to the nearby local supernovae.

(29) Since consecutive expansion epochs of the Universe preceded the current expansion epoch, as proved in this paper, therefore, it is prudent to consider that the current expansion epoch is definitely not the last one; consecutive expansion epochs succeeding the current expansion epoch are also expected. The expansion rate obtained from such consecutive expansion epochs that will succeed the current expansion epoch will also appear to be higher than the expansion rate of the current expansion epoch, again, not because of acceleration, but because the expansion initiated comparatively later.

(30) The study conducted in this paper does not advocate in any way that we are located in a special or privileged position within the Universe. The study conducted in this paper is also not based in any way on inhomogeneous/ anisotropic expansion. The study conducted in this paper clearly states, deals with, emphasises on, and therefore advocates "consecutive expansion epochs of the Universe that preceded the current expansion epoch". As long as there are epochs of consecutive expansion, (one expansion epoch following the next in a consecutive manner) an observer in any expansion epoch will observe expansion attributes that appear similar to cosmic acceleration, that is, "cosmic deceleration that preceded the current epoch of cosmic acceleration" - to an observer in any expansion epoch, preceding expansion epochs will appear to be decelerating, whereas the observer's current epoch of cosmic expansion that happens to succeed the preceding expansion epochs will appear to be accelerating. In reality, however, such observation would be attributed not to deceleration or acceleration, but to consecutive expansion epochs wherein one expansion epoch is followed by the next in a consecutive manner. The expansion rate of preceding expansion epoch appears to be lower (decelerating) as compared to the expansion rate of the expansion epoch that succeeds it - a natural feature of consecutive expansion.

(31) The primary advantage of the study conducted in this paper is that it does not question or doubt the supernovae observations carried out by the Nobel Prize-winning teams - the observations that showed remote supernovae are further away than expected. The study takes into consideration the observations carried out by the Nobel Prize-winning teams and helps providing a robust and a novel explanation why remote supernovae would end up being further away than expected without acceleration.

(32) Given that the apparent transition of Universe's expansion from deceleration to acceleration cannot be explained ("Unfortunately, no obvious breakthrough in our understanding has yet occurred - cosmic acceleration remains the same mystery that it was in 1998" Schmidt (2012)), mysterious and hypothetical dark energy of unknown origin having no explanation in fundamental physics, and then the 120-ordersof-magnitude discrepancy involved while calculating the theoretical value of the energy associated with empty space, we therefore need to consider the study conducted in this paper that perfectly mimics cosmic acceleration based on multiple similarities (replication and reproduction of results) obtained in a single paper while plotting velocity-distance relationship, expansion rate vs. time relationship, expansion factor vs. time relationship, scale factor vs. time relationship, scale factor vs. distance relationship, distance-redshift relationship, and distance modulus vs. redshift relationship, moreover, and most importantly, deceleration parameter $\left(q_{0}\right)$ was also found to be deviating from positive $\left(q_{0}>0\right)$ to increasingly negative values $\left(q_{0}<0\right)$ while using more recent or evolving values of the expansion rate $(H)$

\section{ACKNOWLEDGEMENTS}

I am grateful to the organising committee (Department of Physics, University of Pune) for giving me an opportunity to present my work at the $26^{\text {th }}$ Raman Memorial Conference held at Department of Physics, University of Pune on $14^{\text {th }}$ February, 2020. I am grateful to the Supernova Cosmology Project team for the data pertaining to 580 type Ia supernovae plotted in Figure 1 (Union 2 (Amanullah et al. 2010) and Union 2.1 (Suzuki et al. 2012)). I am grateful to the GOODS (Great Observatories Origins Deep Survey) Treasury program team (joint work conducted by Giavalisco et al. 2004 and Riess et al. 2004) for the high-redshift type Ia supernovae discovered by them through the ACS (Advanced Camera for Surveys) on the Hubble Space Telescope ( 7 of these high-redshift type Ia supernovae have been plotted in Figure 1). I am grateful to Gilliland et al. (1999) for the very high-redshift type Ia supernova discovered by them with WFPC2 (Wide Field and Planetary Camera 2) on the Hubble Space Telescope (1 highredshift type Ia supernova has been plotted in Figure 1). I am grateful to American Physical Society for allowing me to illustrate the Hubble Diagram of SNe Ia in my manuscript (Figure 2; illustrated from Schmidt (2012)). I am grateful to European Southern Observatory (ESO) for allowing me to illustrate the Velocity versus luminosity-distance for type Ia supernovae, $\mathrm{S}-\mathrm{Z}$ clusters and gravitational lens time-delay systems in my manuscript (Figure 4; illustrated from Blanchard et al. (2003)). I am also grateful to the American Institute of Physics for allowing me to illustrate the scale factor versus light-travel-time relationship for type Ia supernovae in my manuscript (Figure 15; illustrated from Perlmutter (2003)).

\section{ORCID iD}

Karan R.Takkhi https://orcid.org/0000-0002-3859-6511 


\section{CONTRIBUTION}

The author (Karan R.Takkhi) participated in the analysis and in writing the paper. The author has also reviewed the manuscript.

\section{COMPETING FINANCIAL INTERESTS}

The author has no competing financial interests.

\section{DATA AVAILABILITY}

Any data that support the findings of this study are included within the article. The data underlying this article (plotting of Figure 1 and additional plots based on Figure 1) were accessed by using the data for 580 type Ia supernovae from the Supernova Cosmology Project (Union 2 and Union 2.1), 7 additional high-redshift type Ia supernovae discovered through the ACS (Advanced Camera for Surveys) on the Hubble Space Telescope from the GOODS (Great Observatories Origins Deep Survey) Treasury program, and 1 additional very high-redshift type Ia supernova discovered with WFPC2 (Wide Field and Planetary Camera 2) on the Hubble Space Telescope).

\section{REFERENCES}

[1] Aguirre A. N., 1999a, ApJ, 512, L19

Dust versus Cosmic Acceleration

[2] Aguirre A. N., 1999b, ApJ, 525, 583

Intergalactic Dust and Observations of Type Ia Supernovae

[3] Amanullah R., et al., 2010, ApJ, 716, 712

Spectra and Hubble Space Telescope Light Curves of Six Type Ia Supernovae at $0.511<z<1.12$ and the Union2 Compilation

[4] Blanchard A., et al., 2003, A\&A, 412, 35

An alternative to the cosmological "concordance model"

[5] Coil A. L., et al., 2000, ApJ, 544, L111

Optical Spectra of Type Ia Supernovae at $z=0.46$ and $z=1.2$

[6] Colin J., et al., 2019, A\&A, 631, L13

Evidence for anisotropy of cosmic acceleration

[7] Drell P. S., et al., 2000, ApJ, 530, 593

Type Ia Supernovae, Evolution, and the Cosmological Constant

[8] Durrer R., 2011, RSPTA, 369, 5102

What do we really know about Dark Energy?

[9] Einstein A., 1917, Sitz. Preuss. Akad. Wiss. Phys.-Math, 142, 87 Cosmological considerations in the general theory of relativity

[10] Freedman W. L., et al., 2001, ApJ, 553, 47

Final Results from the Hubble Space Telescope Key Project to Measure the Hubble Constant

[11] Giavalisco M., et al., 2004, ApJ, 600, L93

The Great Observatories Origins Deep Survey: Initial Results from Optical and Near-Infrared Imaging scovered Aspect that Perfectly Mimics Cosmic Acceleration

[12] Gilliland R. L., et al., 1999, ApJ, 521, 30

High-Redshift Supernovae in the Hubble Deep Field

[13] Hubble E. P., 1929, Proc. Natl. Acad. Sci., 15, 168

$A$ relation between distance and radial velocity among extragalactic nebulae

[14] Leibundgut B., 2001, ARA\&A, 39, 67

Cosmological Implications from Observations of Type Ia Supernovae

[15] Perlmutter S., et al., 1999, ApJ, 517, 565

Measurements of $\Omega$ and $\Lambda$ from 42 High-Redshift Supernovae

[16] Perlmutter S., 2003, Physics Today, 56, 53

Supernovae, Dark Energy, and the Accelerating Universe

[17] Räsänen S., 2011, Class. Quant. Grav., 28, 164008 Backreaction: directions of progress

[18] Riess A. G., et al., 1998, AJ, 116, 1009

Observational Evidence from Supernovae for an Accelerating Universe and a Cosmological Constant

[19] Riess A. G., 2000, PASP, 112, 1284

The Case for an Accelerating Universe from Supernovae

[20] Riess A. G., et al., 2001, ApJ, 560, 49

The Farthest Known Supernova: Support for an Accelerating Universe and a Glimpse of the Epoch of Deceleration

[21] Riess A. G., et al., 2004, ApJ, 607, 665

Type Ia Supernova Discoveries at $z>1$ from the Hubble Space Telescope: Evidence for Past Deceleration and Constraints on Dark Energy Evolution

[22] Riess A. G., 2012, Rev. Mod. Phys., 84, 1165

Nobel Lecture: My path to the accelerating Universe

[23] Sabulsky D. O., et al., 2019, Phys. Rev. Lett., 123, 061102 Experiment to Detect Dark Energy Forces Using Atom Interferometry

[24] Schmidt B. P., 2012, Rev. Mod. Phys., 84, 1151 Nobel Lecture: Accelerating expansion of the Universe through observations of distant supernovae

[25] Sullivan M., et al., 2003, MNRAS, 340, 1057

The Hubble diagram of type Ia supernovae as a function of host galaxy morphology

[26] Suzuki N., et al., 2012, ApJ, 746, 85

The Hubble Space Telescope Cluster Supernova Survey. V. Improving the Dark-energy Constraints above $z>1$ and Building an Early-type-hosted Supernova Sample

[27] Zehavi I., et al., 1998, ApJ, 503, 483

A Local Hubble Bubble from Type Ia Supernovae?

[28] https://www.nobelprize.org/uploads/2018/06/popularphysicsprize2011-1.pdf

Written in the stars 